\title{
Use of sediment source fingerprinting to assess the role of subsurface erosion in the supply of fine sediment in a degraded catchment in the Eastern Cape, South Africa
}

\author{
Munyaradzi Manjoro $^{1 *}$, Kate Rowntree ${ }^{2}$, Vincent Kakembo ${ }^{3}$, Ian Foster ${ }^{2,4}$, Adrian L. Collins ${ }^{5}$
}

\author{
*Corresponding author \\ ${ }^{1}$ Department of Geography and Environmental Sciences, North-West University, Mafikeng Campus, \\ Private Bag X2046, Mmabatho 2735, South Africa, Tel: +27 (0)18 389 2357, Fax: +27 (0)18 389 \\ 2377, email: Munyaradzi.Manjoro@nwu.ac.za, \\ ${ }^{2}$ Department of Geography, Rhodes University, Brosty Rd, PO Box 94, Grahamstown, 6140, South Africa, \\ email: K.Rowntree@ru.ac.za \\ ${ }^{3}$ Geosciences Department, Nelson Mandela Metropolitan University, Port Elizabeth, 6031, South Africa, email: \\ Vincent.Kakembo@nmmu.ac.za \\ ${ }^{4}$ Department of Environmental and Geographical Sciences, University of Northampton, Northampton NN2 \\ 6JD, UK, email: Ian.Foster@northampton.ac.uk \\ ${ }^{5}$ Department of Sustainable Soil and Grassland Systems, Rothamstead Research, North Wyke, EX20 2SB, UK, \\ email: Adrian.Collins@Rothamsted.ac.uk
}

\begin{abstract}
It is important to estimate the relative importance of surface and subsurface sources of sediment to help identify the related erosion process and assist in the design and targeting of rehabilitation measures. Sediment source fingerprinting has been successfully deployed to provide information on the surface and subsurface sources of sediment in many catchments around the world. However, there is still scope to re-examine some of the major assumptions of the technique with reference to the number of fingerprint properties used in the model, number of model iterations and the potential uncertainties of using more than one sediment core collected from the same floodplain sink. We investigated the role of subsurface erosion in the supply of fine sediment to two sediment cores collected from a floodplain in a small degraded catchment in the Eastern Cape, South Africa. The results showed that increasing the number of individual fingerprint properties in the composite signature did not improve the model goodness-of-fit. This is still a much debated issue in sediment source fingerprinting. To test the goodness-of-fit further, the model repeat iterations were increased
\end{abstract}


from 5000 to 30000 . However, this did not reduce uncertainty ranges in modelled source proportions nor improve the model goodness-of-fit. However, the estimated sediment source contributions were not consistent with the available published data on erosion processes in the study catchment. The temporal pattern of sediment source contributions predicted for the two sediment cores was very different despite the cores being collected in close proximity from the same floodplain. This highlights some of the potential limitations associated with using floodplain cores to reconstruct catchment erosion processes and associated sediment source contributions. For the source tracing approach in general, the findings here suggest the need for further investigations into uncertainties related to the number of fingerprint properties included in un-mixing models. The findings support the current widespread use of $\leq 5000$ model repeat iterations for estimating the key sources of sediment samples.

\section{Key words}

sediment source fingerprinting, uncertainty analysis, Eastern Cape, gully erosion, mass balance modelling 


\section{Introduction}

Although some research in a range of environments globally suggests that gully erosion represents an important sediment source (Wallbrink et al., 1996; Wasson et al., 1996; Wilkinson et al. 2013), it is still a much debated issue (Govers and Poesen, 1988; Vente and Poesen, 2005). For example, Wallbrink et al., (1996) found that 90\% of the suspended sediment load in the lower Murrumbidgee River, Australia, was derived from subsurface sources and gullies in particular. Wasson et al. (1996) noted that much of the sediment in Australian rivers is derived from gully sources and estimated that the presence of gullies increased sediment emissions by a factor of 10 . Poesen et al. (2002) reported that gully erosion represents an important sediment source in dry land environments contributing, on average, $50 \%$ to $80 \%$ of overall sediment production. The effect of gully erosion on sediment generation and catchment scale sediment delivery is, however, dependent on the ability of the gullies to route sediment efficiently into fluvial systems (Foster et al., 2012; Fuller and Marden, 2010). This is best described by looking at the degree of coupling (Harvey, 2001) or connectivity (Fryirs et al., 2007), between sediment producing areas and channel systems. Clearly, the role of gully erosion as a catchment sediment source may not be generalized and needs a case by case analysis.

Gully erosion is a major feature of Eastern Cape landscapes in South Africa (Boardman et al., 2003; Kakembo et al., 2009; Key-Bright and Boardman, 2009; Le Roux and Sumner, 2011). Where the problem is most serious, large expanses of land are heavily dissected forming extensive so-called 'badlands'. Most of these are deeply incised into colluvial hill slopes and weathered shale bedrock (Boardman and Foster, 2008; Kakembo et al., 2009). Studies have found that gullies differ in size in response to different factors. For example, Dollar and Rowntree (1995) measured gullies up to $22 \mathrm{~m}$ wide and $13 \mathrm{~m}$ deep in cultivated fields in the Bell River catchment. Hanvey et al. (1991) described gullies $20 \mathrm{~m}$ wide and $16 \mathrm{~m}$ deep on a fossil dune complex in the east coast of South Africa. Gullies eroding on bedrock in the arid landscapes of Karoo region of the Eastern Cape in South Africa have been known to reach depths of $5 \mathrm{~m}$ and widths of over $20 \mathrm{~m}$ and even deeper in valley bottoms (Boardman and Foster, 2008). Both short and medium term rates of gully erosion have been estimated in the Eastern Cape province. For example, Boardman et al., (2010) reported gully erosion rates of between $32.3 \mathrm{t} \mathrm{ha}^{-1} \mathrm{y}^{-1}$ to $136 \mathrm{t} \mathrm{ha}^{-1} \mathrm{y}^{-1}$ in the Karoo. Other studies have estimated gully erosion rates in terms of changes in spatial extent on the basis of aerial photographs. Dollar and Rowntree (1995) noted an increase in the total gully length of $69 \%$ between 1952 and 1975 in the Bell River catchment, Eastern Cape and by a further $169 \%$ up to 1991 . Vetter (2007) noted a substantial increase in erosion 
from 1950 to 1995 in the Sterkspruit District with more than 50\% of the surface area affected by sheet, rill or gully erosion by 1995 .

Investigations have reached varied conclusions in relation to the linkages between gully erosion and high sediment yields in catchments of the Eastern Cape province. Foster et al. $(2007 ; 2012)$ reported that rather than being a major source of contemporary sediment, gullies only provide connectivity between the eroding upper section and the river systems in some of the catchments in the Karoo region. This was consistent with Keay-bright and Boardman (2006) who reported that gully and badland expansion in the same region has slowed down, stabilized and even decreased. However, Rowntree and Foster (2012) reported that regardless of the above observation, some gullies continue to erode at high rates and continue acting as 'partial areas' for sediment contribution to river systems even when the badland area is relatively small in size.

It is known that sheet and rill (i.e. top soil source) and gully and bank erosion (i.e. subsoil sources) are the major sources of the fine-grained bed and suspended load in many river systems (Wethered et al., 2015). However, the contributions of topsoil and subsoil vary from catchment to catchment undermining generalisations. Knowledge of the relative importance of surface and subsurface sources of sediment helps identify the main erosion process mobilizing sediment and thus provides assistance in the design and targeting of rehabilitation measures to reduce downstream sediment loads and associated off-site impacts. This information may also be important to understand catchment sediment delivery processes and the degree of lateral and longitudinal (dis)connectivity of the catchment sediment cascade (Fryirs et al., 2007; Koiter et al., 2013; Smith and Dragovich 2008; Wethered et al., 2015). Given the uncertainty surrounding the role of gullies as a sediment source in South African catchments, this study explores the issue further by focussing on a catchment located in the Ngqushwa Local Municipality, Eastern Cape, South Africa, where severe soil erosion resulting from land use change has led to landscape dysfunction (Kakembo et al., 2009) and excessive sedimentation in the local stream channels (Kakembo and Rowntree, 2003). Sediment source fingerprinting potentially offers an opportunity to reconstruct historical sediment source dynamics in terms of surface and subsurface contributions on the basis of the sediment signatures preserved in floodplain sediment cores (Collins et al., 1997b; Collins et al., 2010a; Owens et al., 1999).

In the context of the above background, this paper specifically aimed to: 1) examine the potential impact of numbers of properties in optimised signatures, number of model iterations and the 
use of replicate depositional sink sampling on the modelled sediment source estimates generated, and; 2) use sediment source fingerprinting to assess the extent to which the supply of fine-grained sediment in the study catchment is dominated by gully and stream bank erosion.

\section{The study area}

The study catchment $\left(90 \mathrm{~km}^{2}\right)$ is located south of the town of Peddie, Eastern Cape province, South Africa (Figure 1). Topography is generally undulating, rising from sea level on the coast to about $365 \mathrm{~m}$ above sea level in the north. Slopes rise steeply ( $>10$ degrees) alongside major stream channels. The catchment is dominated by shale and arenite of the Ecca Group and Karoo Supergroup (PermianTriassic). The Ecca Group shale weathers to form highly erodible soils (Mills and Cowling, 2006). Greyish brown shallow litholic soils of the Mispah and Glenrosa Form (Entisols and Inceptisols in Soil Taxonomy) which are dominant in the catchment, are typically low in organic matter content and sometimes with high sodium content (Kakembo and Rowntree, 2003). Severe soil erosion which ranges from sheet and inter-rill erosion in grazing lands to gully erosion in abandoned cultivated lands has been well documented in the study catchment (see Kakembo, 2009; Kakembo and Rowntree, 2003; Kakembo et al., 2009; Manjoro et al., 2012a). Gully erosion is mostly predominant in lower slope positions.

The average annual rainfall is $491 \mathrm{~mm}$ and is bi-modally distributed, with peaks in October or November and March or April (ARC 2010). Vegetation is heavily modified by human activity and only a mosaic of the degraded thicket vegetation exists in patches within the study catchment and along some riparian and coastal corridors. The main land cover categories are grassland, mostly used for extensive grazing, and areas of woody shrub vegetation cover, most of which is Pteronia Incana found mainly in abandoned cultivated lands and some grazing lands. Some cultivation (mainly pine apples and maize) takes place in the north-east and north-west of the study catchment. 


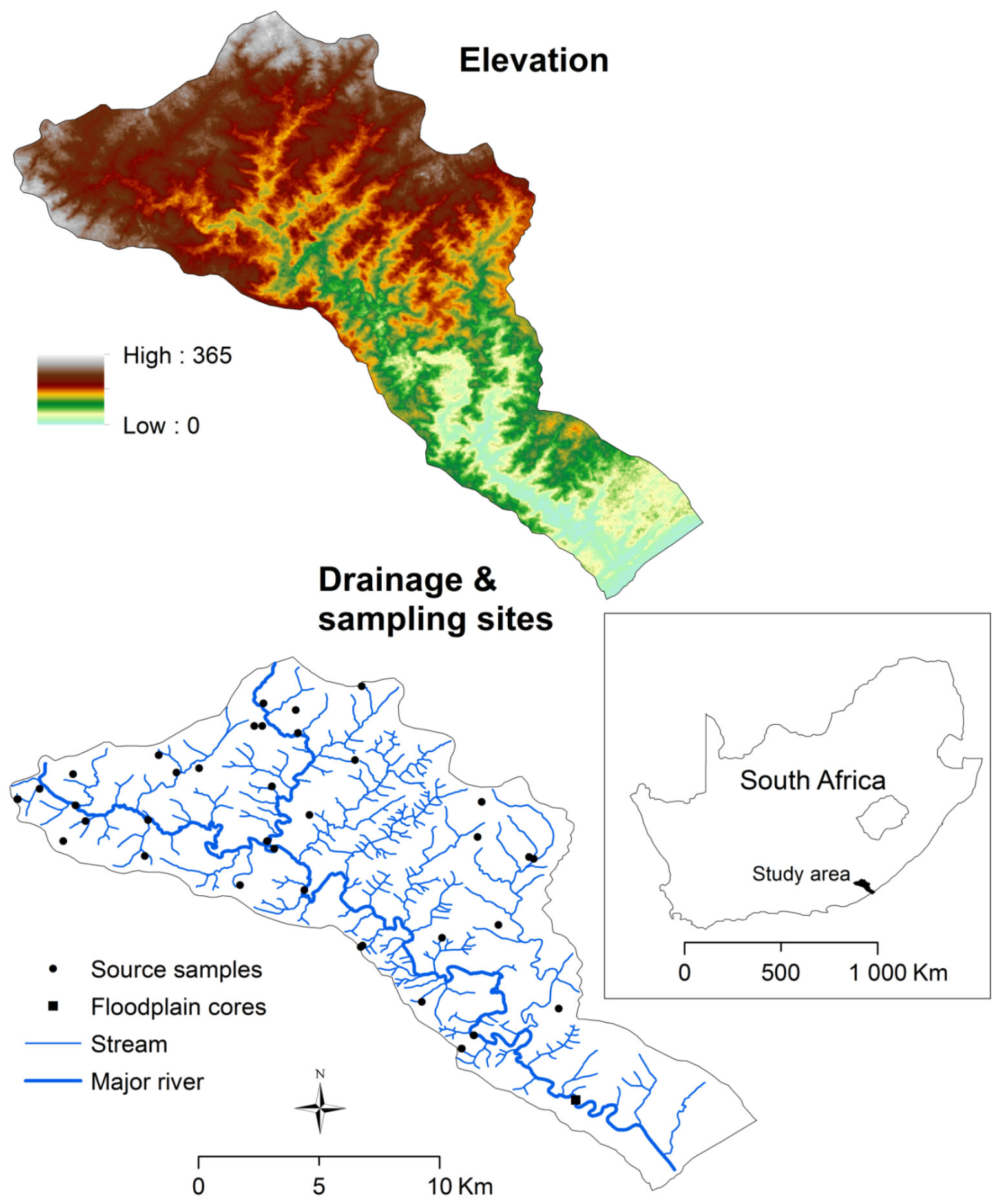

Figure 1. Study area. 


\section{The approach}

Sediment source fingerprinting is increasingly being used to quantify the contribution of different sediment sources (different land uses, topsoil/subsoil, geological sub-areas, sub-catchments), evaluate catchment soil erosion dynamics and develop management plans to tackle sediment-related problems including reservoir siltation and sediment-bound pollutant transfers (Gruszowski et al., 2003; Rowan et al., 2012; Walling et al., 2008). The technique relies upon the link between sediment properties and those of its principal sources (Foster and Lees, 2000; Walling, 2005). Sediment source fingerprinting has been used successfully to identify and apportion the relative importance of topsoil and subsoil in different environmental settings (Evrard et al., 2013; Hughes et al., 2009; Smith and Dragovich 2008; Wallbrink and Olley 2004; Weathered et al., 2015; Wilkinson et al., 2013). On the basis of the previous reported success in using sediment fingerprinting to apportion topsoil and subsoil sediment sources, this study investigated the extent to which subsoil is the main sediment source in the study catchment. Sediment source fingerprinting was used to discriminate and apportion the surface and subsurface sources of fine sediment deposited in a major floodplain in the study catchment and to help infer the main erosion processes mobilizing sediment in the catchment for the purpose of informing targeted management.

\subsection{Sampling of potential sediment sources and the floodplain sink}

According to Mukundan et al. (2012), an effective strategy for sampling both sources and sediment is key to developing meaningful sediment source ascription results with less uncertainty. Sampling of the potential sediment sources was stratified to generate information on the major observed processes mobilizing fine sediment in the study catchment; that is, whether the processes are surface (sheet and inter-rill erosion) or subsurface (rill or gully or river bank erosion). Topsoil samples were collected regardless of land use or geology on the basis of visual evidence of erosion. For sediment source fingerprinting purposes, researchers have used various representative depths for surface soil samples ranging from the top $0.5 \mathrm{~cm}$ (Minella et al., 2008; Wallbrink et al., 1998) or 2-3 cm (Hughes et al., 2009; Laceby and Olley, 2015; Wethered et al., 2015) and up to $5 \mathrm{~cm}$ (Devereux et al., 2010). In this study, all topsoil samples $(\mathrm{n}=17)$ were collected from the upper $3 \mathrm{~cm}$ of the soils. This sampling depth ensured that only surface material likely to be mobilized by surface processes was sampled (cf. Collins et al., 1997; Hancock and Pietsch, 2008; Hughes et al., 2009; Walling, 2005). Roddy (2010) reported about various sediment source material sampling strategies ranging from 
randomised to targeted approaches. Targeted sampling of actively eroding sites was the main strategy used. This sampling strategy was adopted to ensure that only those eroding areas with clear connectivity to river channel systems were sampled. Manjoro et al. (2012b) noted cases of slopechannel disconnectivity in the upper parts of the study catchment. This is important since previous research has shown that assumptions about source-channel connectivity can have considerable impact on uncertainties associated with sediment source fingerprinting (Koiter et al., 2013; Martínez-Carreras et al., 2009). Four to five topsoil samples (individual sample size typically $\geq 500 \mathrm{~g}$ ) were collected in the vicinity of each sampling point covering a radius of about 30 metres and put in separate sample bags. After oven drying $\left(40{ }^{\circ} \mathrm{C}\right)$ back in the laboratory, an equal mass $(250 \mathrm{~g})$ from each of the individual samples was combined and homogenized to make one composite sample for each catchment sampling point $(\mathrm{n}=33)$. Compositing samples for subsequent laboratory analyses helps make the source material samples representative of the potential spatial variability within the individual sources (Minella et al., 2008; Russell et al., 2001; Wethered et al., 2015), while reducing laboratory analysis costs. Subsoil samples $(n=16)$ were obtained directly from actively eroding gully sidewalls, head cuts and exposed river channel banks and comprised material from the full vertical extent of the exposures by scraping soil/regolith from erosion scars (Collins et al., 2010a). Global positioning system (GPS) coordinates of all sampling points were recorded and plotted on a map (Figure 1).

To provide sediment for the source tracing exercise, two sediment cores were collected $35 \mathrm{~m}$ apart in a floodplain of the Mgwalana River, the main stream of the study catchment (Figure 1). The selected sampling sites were located in the mid-section of the inside of a meander loop and were informed by visual observations and reports on inundation patterns during overbank events. The first site was located $5 \mathrm{~m}$ from the river bank and Site 2 was located $35 \mathrm{~m}$ away. The floodplain cores were retrieved using a manually operated Eijkelkamp corer with a plastic lined chamber of $5 \mathrm{~cm}$ diameter and $30 \mathrm{~cm}$ depth. At Site 1, it was only possible to collect the sediment core up to $103 \mathrm{~cm}$ depth, whereas at Site 2, the core was collected up to a depth of $213 \mathrm{~cm}$. The bottom part of this core was not suitable for tracing as it consisted mostly of coarse sediment. The collection of two floodplain cores provided an opportunity to examine uncertainties in sediment source estimates associated with using two sediment profiles collected from the same floodplain sink.

\subsection{Laboratory methods}


All source and floodplain core (sectioned at $4 \mathrm{~cm}$ depth intervals) sediment samples were manually disaggregated using a mortar and pestle and dry sieved to isolate the fine-grained fraction $(<63 \mu \mathrm{m})$. It is assumed that this size fraction is representative of fluvial suspended sediment (Walling et al., 2000) transported through most river systems. Sediment source fingerprinting involves measuring sediment properties that are capable of distinguishing different catchment sediment sources (Collins et al., 1996; Olley and Caitcheon 2000). Mineral-magnetic and geochemical analyses were undertaken on all soil and sediment samples and caesium-137 $\left(\mathrm{Cs}^{137}\right)$ activity was measured on selected sediment samples for the purpose of dating one of the floodplain sediment cores. Standard mineral-magnetic measurements (Dearing 1999; Walden 1999) were undertaken on all samples. These included low ( $\chi \mathrm{lf})$ and high $(\chi \mathrm{hf})$ frequency magnetic susceptibility, anhesteric remanant magnetisation (ARM), soft isothermal remanent magnetisation (IRM-100mT) and saturation isothermal remanent magnetisation $\left(\mathrm{IRM}_{1000 \mathrm{mT}}\right)(\mathrm{SIRM})$. Various other mineral-magnetic parameters were derived from the measurements (e.g. frequency dependent susceptibility $(\chi \mathrm{fd})$, susceptibility of ARM ( $\chi \mathrm{ARM}$ and hard isothermal remanent magnetisation (HIRM)). Organic matter content was estimated by loss-onignition (LOI) at low temperature $\left(550^{\circ} \mathrm{C}, 3\right.$ hours) (Heiri et al., 2001). Inductively coupled plasma mass spectrometry (ICP-MS) was undertaken on all samples following microwave oven-assisted digestion using aqua regia $\left(\mathrm{HCl}: \mathrm{HNO}_{3}, 3: 1, \mathrm{v} / \mathrm{v}\right)$ (USEPA, 2007). This yielded concentrations of various rare earth ( $\mathrm{La}, \mathrm{Ce}, \mathrm{Eu}, \mathrm{Sm}, \mathrm{Lu}, \mathrm{Tb}, \mathrm{Yb}$ ), trace (As, Co, Ba, Cr, Cs, Th, Zn, Cd, Mn, Cu, Ni, $\mathrm{Pb}, \mathrm{Tl}, \mathrm{V}, \mathrm{Li}, \mathrm{B}, \mathrm{Sc}, \mathrm{Ga}, \mathrm{Rb}, \mathrm{Sr}, \mathrm{Y}, \mathrm{Ru}, \mathrm{Rh}, \mathrm{Pd}, \mathrm{Pr}, \mathrm{Nd}, \mathrm{Gd}, \mathrm{Dy}, \mathrm{Ho}, \mathrm{Er}, \mathrm{Tm}, \mathrm{Re}, \mathrm{Os}, \mathrm{Ir}, \mathrm{Pt}, \mathrm{Au}, \mathrm{U})$ and major (Fe, K, Na, Al, Ca, Mg) elements which were assessed for sediment source tracing purposes.

Mineral-magnetic, geochemical and radionuclide signatures are often controlled by the particle size distribution (He and Walling, 1996; Horowitz, 1991; Owens et al., 2000). To enable correction for the potential effects of particle size differences between source and sediment samples on the corresponding signatures, the absolute grain size composition of all samples was determined using a sedigraph (Micromeritics SediGraph III 5120, USA) following sample pre-treatment with hydrogen peroxide $\left(\mathrm{H}_{2} \mathrm{O}_{2}\right)(30 \% \mathrm{v} / \mathrm{v})$ and boiling at $100{ }^{\circ} \mathrm{C}$ on a hotplate to eliminate organic matter (Walling et al., 2000). This was followed by chemical dispersion with sodium hexametaphosphate and 5 minutes in an ultrasonic bath before analysis. Estimates of specific surface area $\left(\mathrm{SSA} ; \mathrm{m}^{2} \mathrm{~g}^{-1}\right)$ for each individual sample were obtained from the particle size measurement software, based on cumulative particle size distributions of the sample and assuming spherical particles (Collins et al., 1998). ${ }^{137} \mathrm{Cs}$ activity was measured by a high-resolution, high purity germanium (HPGe) 'well' detector. Foster et al. (2005) provide details of energy and efficiency calibration methods, and of quality control used for 
such analysis. Count times were typically 172000 to 216000 seconds, with the counting errors reported to $2 \sigma$ (i.e. $95 \%$ confidence limits).

Figure 2 presents the ${ }^{137} \mathrm{Cs}$ depth profile of the sediment core extracted at Site 2. It can be observed that there is no clearly defined peak in the profile and some of the depth incremental samples in the upper half have ${ }^{137} \mathrm{Cs}$ activity below detection limits. This normally reflects input of sediment derived from areas with low or no ${ }^{137} \mathrm{Cs}$ activity such as stream bank and gully erosion sites (Amos et al., 2009; Wallbrink et al., 1996). As such, the accuracy of the ${ }^{137}$ Cs date of first detection (Leslie and Hancock, 2008) or peak fallout, both of which are used as chronological markers for dating purposes is reduced (Amos et al., 2009; Hughes et al.,2009). Thus, without a reliable detailed chronology an attempt to investigate specific temporal variations in sediment source was considered unfeasible. The ${ }^{137} \mathrm{Cs}$ activity profile was, however, used to obtain a general temporal framework for the reconstruction of both contemporary and historical surface and subsurface sediment source contributions in the study area, by dividing the sediment core into three time frames. The first $8 \mathrm{~cm}$ represented contemporary sediment (2000-2010). The rest of the older deposited sediment was divided into two: the mid-section $(8-110 \mathrm{~cm} ; 1958-2000)$ where most of the sediment had detectable ${ }^{137} \mathrm{Cs}$ and the bottom section $(110$ $200 \mathrm{~cm}$ ) with no detectable ${ }^{137} \mathrm{Cs}$ content. The bottom-most layer with the detectable ${ }^{137} \mathrm{Cs}$ is normally allocated to 1955 in the southern hemisphere (Leslie and Hancock, 2008). However, studies have demonstrated that ${ }^{137} \mathrm{Cs}$ is not likely to be measurable in South African environments before 1958 (Rowntree and Foster, 2012). Thus, the first occurrence of ${ }^{137} \mathrm{Cs}$ in the sediment core was ascribed to 1958. Assuming no substantial post-depositional down-profile mobility of the ${ }^{137} \mathrm{Cs}$, we ascribed 1958 to the depth of $110 \mathrm{~cm}$. Sediment below a depth of $110 \mathrm{~cm}$ was therefore taken to pre-date 1958 . 


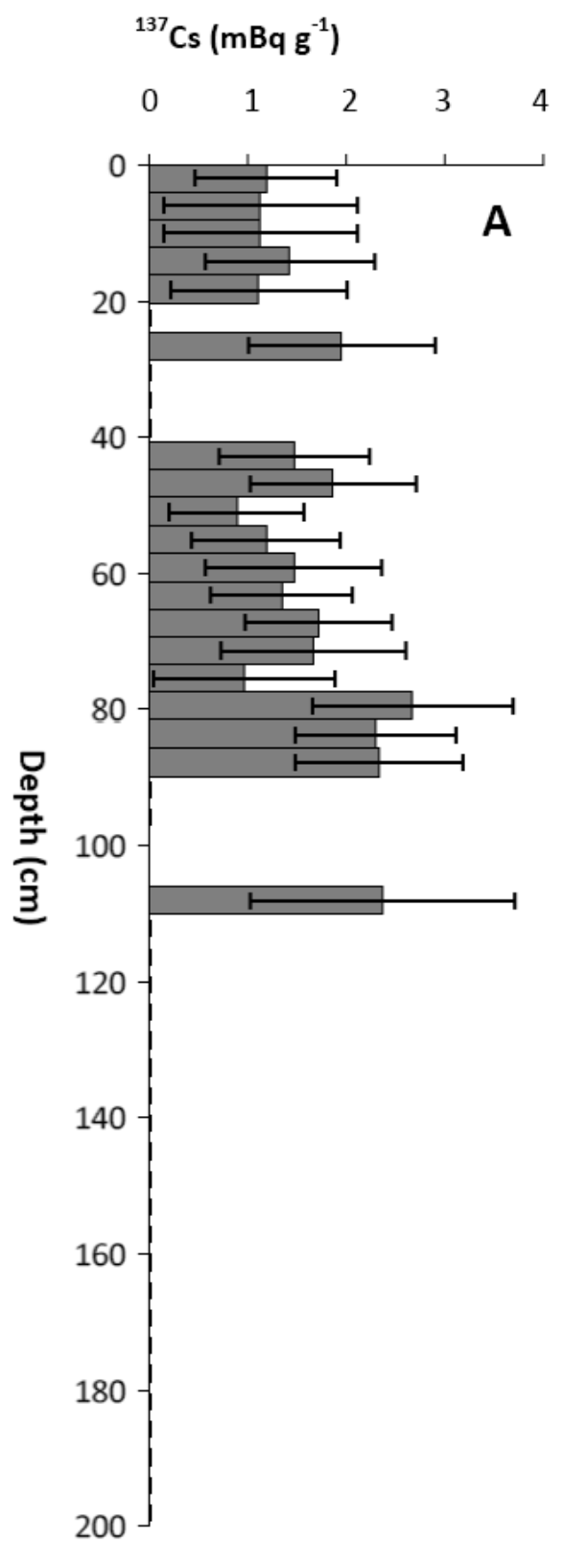

Figure $2 .{ }^{137} \mathrm{Cs}$ depth profile for the floodplain core collected at site 2 . The error bars represent measurement precision (95\% confidence limits). 


\subsection{Statistical selection of composite fingerprints to discriminate surface and subsurface catchment sediment sources}

As a first step, it was necessary to test normality of the fingerprint datasets for each sediment source category (Collins et al., 2012a). Lilliefor's Test (Henderson, 2006; Lilliefors, 1969) was used with a null hypothesis that all properties are random and come from a normal distribution. The Lilliefor's Test represents an adaptation of the Kolmogorov-Smirnov test and provides a two sided goodness-of-fit procedure in situations where the fully specified null population for each fingerprint property is unknown, thereby requiring the estimation of its parameters using the significance of comparison at $p=\leq 0.05$. During the application of the Lilliefors test, the sample mean and standard deviation were used to represent the corresponding values for the benchmark population against which the measured fingerprint property data were compared. Table 1 shows that the null hypothesis that the samples came from a normal distribution was not satisfied in all cases, suggesting some tracers were not normally distributed.

The next step was the tracer mass conservation test (Collins et al., 2013). In situ postdepositional change in sediment properties can be a serious problem for applying sediment source fingerprinting using historical sediment (Collins et al., 1997b; D’Haen et al., 2012; Koiter et al., 2013; Macklin et al., 1994). Thus, it was necessary to assess whether the ranges of values for individual tracers associated with the sediment comprising each floodplain core were within the corresponding range of values measured for the study catchment potential sources. This range test was undertaken using the tracer parameter source end member and sediment medians and a robust scaling estimator Qn (Collins et al., 2012a; Rousseeuw and Croux, 1993) with a correction factor for absolute particle size differences between the floodplain core sediment and the source samples. The median and Qn are considered more appropriate where a small number of samples have been collected to characterize potential sources (Stone et al.,2014) and are more robust in estimating tracer property parameter location and scale rather than the conventional parameters (mean and standard deviation) in situations where some properties are not uniformly distributed. The latter are more prone to be influenced by outliers (Collins et al., 2012a, 2010; Tabachnick and Fidell, 1996). Because the Lilliefors test results (Table 1) clearly indicated that not all tracers are normally distributed, robust as opposed to parametric statistics were used in further analyses. The range test was applied separately to each floodplain core section. 
Table 1. The results of the Lilliefors test.

Site 1

\begin{tabular}{|c|c|c|c|}
\hline Surface & & Subsurfac & \\
\hline Property & $\mathrm{p}$-Value & Property & $\mathrm{p}$-Value \\
\hline $\mathrm{Li}$ & 0.50 & $\mathrm{Li}$ & $0.03^{*}$ \\
\hline B & 0.27 & $\mathrm{~B}$ & 0.50 \\
\hline $\mathrm{Na}$ & $0.00^{*}$ & $\mathrm{Na}$ & $0.00^{*}$ \\
\hline $\mathrm{Mg}$ & $0.04^{*}$ & $\mathrm{Mg}$ & 0.25 \\
\hline $\mathrm{Al}$ & 0.17 & $\mathrm{Al}$ & 0.14 \\
\hline $\mathrm{K}$ & 0.10 & $\mathrm{~K}$ & 0.50 \\
\hline $\mathrm{Ca}$ & 0.12 & $\mathrm{Ca}$ & 0.50 \\
\hline $\mathrm{Sc}$ & 0.50 & $\mathrm{Sc}$ & 0.34 \\
\hline $\mathrm{V}$ & 0.50 & $\mathrm{~V}$ & 0.20 \\
\hline $\mathrm{Cr}$ & $0.00^{*}$ & $\mathrm{Cr}$ & 0.41 \\
\hline $\mathrm{Mn}$ & 0.01 & $\mathrm{Mn}$ & 0.47 \\
\hline $\mathrm{Fe}$ & 0.29 & $\mathrm{Fe}$ & 0.41 \\
\hline Co & 0.09 & Co & 0.15 \\
\hline $\mathrm{Ni}$ & $0.00^{*}$ & $\mathrm{Ni}$ & $0.01^{*}$ \\
\hline $\mathrm{Cu}$ & 0.19 & $\mathrm{Cu}$ & 0.23 \\
\hline $\mathrm{Zn}$ & 0.50 & $\mathrm{Zn}$ & 0.27 \\
\hline $\mathrm{Ga}$ & 0.20 & $\mathrm{Ga}$ & 0.50 \\
\hline As & $0.00^{*}$ & As & 0.34 \\
\hline $\mathrm{Rb}$ & 0.50 & $\mathrm{Rb}$ & 0.34 \\
\hline $\mathrm{Sr}$ & $0.03^{*}$ & $\mathrm{Sr}$ & 0.19 \\
\hline $\mathrm{Y}$ & 0.05 & $\mathrm{Y}$ & 0.50 \\
\hline $\mathrm{Pd}$ & 0.46 & $\mathrm{Pd}$ & 0.25 \\
\hline $\mathrm{Cd}$ & 0.14 & $\mathrm{Cd}$ & 0.26 \\
\hline $\mathrm{Cs}$ & 0.50 & $\mathrm{Cs}$ & $0.01^{*}$ \\
\hline $\mathrm{Ba}$ & $0.01^{*}$ & $\mathrm{Ba}$ & 0.12 \\
\hline $\mathrm{La}$ & 0.49 & $\mathrm{La}$ & 0.50 \\
\hline $\mathrm{Ce}$ & 0.06 & $\mathrm{Ce}$ & 0.50 \\
\hline $\operatorname{Pr}$ & 0.50 & $\operatorname{Pr}$ & 0.50 \\
\hline $\mathrm{Nd}$ & 0.50 & $\mathrm{Nd}$ & 0.50 \\
\hline $\mathrm{Sm}$ & 0.19 & $\mathrm{Sm}$ & 0.50 \\
\hline $\mathrm{Eu}$ & 0.27 & $\mathrm{Eu}$ & 0.50 \\
\hline $\mathrm{Gd}$ & 0.08 & $\mathrm{Gd}$ & 0.31 \\
\hline $\mathrm{Tb}$ & $0.00^{*}$ & $\mathrm{~Tb}$ & 0.30 \\
\hline Dy & 0.05 & Dy & 0.17 \\
\hline Ho & 0.10 & Ho & 0.16 \\
\hline $\mathrm{Er}$ & 0.05 & $\mathrm{Er}$ & 0.35 \\
\hline $\mathrm{Tm}$ & 0.07 & $\mathrm{Tm}$ & 0.08 \\
\hline $\mathrm{Yb}$ & 0.12 & $\mathrm{Yb}$ & 0.07 \\
\hline
\end{tabular}

Site 2

\begin{tabular}{|c|c|c|c|}
\hline \multirow{2}{*}{$\begin{array}{l}\text { Surface } \\
\text { Property }\end{array}$} & \multicolumn{3}{|c|}{ Subsurface } \\
\hline & p-Value & Property & p-Value \\
\hline $\mathrm{Li}$ & 0.50 & $\mathrm{Li}$ & $0.02^{*}$ \\
\hline $\mathrm{B}$ & 0.41 & $\mathrm{~B}$ & 0.50 \\
\hline $\mathrm{Na}$ & $0.00^{*}$ & $\mathrm{Na}$ & $0.00^{*}$ \\
\hline $\mathrm{Mg}$ & $0.02^{*}$ & $\mathrm{Mg}$ & 0.12 \\
\hline $\mathrm{Al}$ & 0.26 & $\mathrm{Al}$ & 0.31 \\
\hline $\mathrm{K}$ & $0.04^{*}$ & $\mathrm{~K}$ & 0.50 \\
\hline $\mathrm{Ca}$ & 0.10 & $\mathrm{Ca}$ & 0.50 \\
\hline $\mathrm{Sc}$ & 0.50 & $\mathrm{Sc}$ & 0.40 \\
\hline $\mathrm{V}$ & 0.50 & $\mathrm{~V}$ & 0.16 \\
\hline $\mathrm{Cr}$ & $0.00^{*}$ & $\mathrm{Cr}$ & 0.23 \\
\hline $\mathrm{Mn}$ & 0.03 & $\mathrm{Mn}$ & 0.27 \\
\hline $\mathrm{Fe}$ & 0.50 & $\mathrm{Fe}$ & 0.23 \\
\hline Co & $0.04^{*}$ & $\mathrm{Co}$ & 0.28 \\
\hline $\mathrm{Ni}$ & $0.00^{*}$ & $\mathrm{Ni}$ & $0.00^{*}$ \\
\hline $\mathrm{Cu}$ & 0.20 & $\mathrm{Cu}$ & 0.46 \\
\hline $\mathrm{Zn}$ & 0.50 & $\mathrm{Zn}$ & 0.13 \\
\hline $\mathrm{Ga}$ & 0.20 & $\mathrm{Ga}$ & 0.50 \\
\hline As & $0.00^{*}$ & As & 0.18 \\
\hline $\mathrm{Rb}$ & 0.37 & $\mathrm{Rb}$ & 0.40 \\
\hline $\mathrm{Sr}$ & $0.01^{*}$ & $\mathrm{Sr}$ & 0.25 \\
\hline $\mathrm{Y}$ & $0.02^{*}$ & $\mathrm{Y}$ & 0.50 \\
\hline $\mathrm{Ru}$ & 0.32 & $\mathrm{Ru}$ & 0.20 \\
\hline $\mathrm{Rh}$ & $0.00^{*}$ & $\mathrm{Rh}$ & $0.00^{*}$ \\
\hline $\mathrm{Pd}$ & 0.42 & $\mathrm{Pd}$ & 0.37 \\
\hline $\mathrm{Cd}$ & 0.06 & $\mathrm{Cd}$ & 0.34 \\
\hline Cs & 0.50 & $\mathrm{Cs}$ & $0.02^{*}$ \\
\hline $\mathrm{Ba}$ & $0.03^{*}$ & $\mathrm{Ba}$ & 0.07 \\
\hline $\mathrm{La}$ & 0.50 & $\mathrm{La}$ & 0.50 \\
\hline $\mathrm{Ce}$ & 0.15 & $\mathrm{Ce}$ & 0.50 \\
\hline $\operatorname{Pr}$ & 0.50 & $\operatorname{Pr}$ & 0.50 \\
\hline $\mathrm{Nd}$ & 0.50 & $\mathrm{Nd}$ & 0.50 \\
\hline $\mathrm{Sm}$ & 0.11 & $\mathrm{Sm}$ & 0.50 \\
\hline $\mathrm{Eu}$ & 0.13 & $\mathrm{Eu}$ & 0.41 \\
\hline $\mathrm{Gd}$ & 0.03 & $\mathrm{Gd}$ & 0.30 \\
\hline $\mathrm{Tb}$ & $0.02^{*}$ & $\mathrm{~Tb}$ & 0.21 \\
\hline Dy & $0.02^{*}$ & Dy & 0.40 \\
\hline Ho & $0.04^{*}$ & Ho & 0.20 \\
\hline $\mathrm{Er}$ & 0.11 & $\mathrm{Er}$ & 0.25 \\
\hline
\end{tabular}




\begin{tabular}{|c|c|c|c|c|c|c|c|}
\hline $\mathrm{Lu}$ & 0.50 & $\mathrm{Lu}$ & 0.18 & $\mathrm{Tm}$ & $0.03^{*}$ & $\mathrm{Tm}$ & 0.06 \\
\hline Os & $0.00^{*}$ & Os & 0.01 & $\mathrm{Yb}$ & 0.20 & $\mathrm{Yb}$ & 0.05 \\
\hline $\mathrm{Tl}$ & 0.27 & $\mathrm{Tl}$ & 0.00 & $\mathrm{Lu}$ & 0.50 & $\mathrm{Lu}$ & 0.14 \\
\hline $\mathrm{Pb}$ & 0.28 & $\mathrm{~Pb}$ & 0.31 & $\mathrm{Re}$ & $0.04^{*}$ & $\mathrm{Re}$ & $0.00^{*}$ \\
\hline Th & 0.14 & Th & 0.29 & Os & $0.00^{*}$ & Os & $0.03^{*}$ \\
\hline $\mathrm{U}$ & 0.35 & $\mathrm{U}$ & 0.13 & Ir & $0.01^{*}$ & Ir & 0.15 \\
\hline Xlf & 0.12 & Xlf & 0.16 & $\mathrm{Pt}$ & 0.03 & $\mathrm{Pt}$ & 0.08 \\
\hline $\mathrm{Xfd}$ & 0.50 & Xfd & 0.14 & $\mathrm{Au}$ & 0.22 & $\mathrm{Au}$ & 0.40 \\
\hline ARM & 0.50 & ARM & $0.04^{*}$ & $\mathrm{Tl}$ & 0.34 & $\mathrm{Tl}$ & $0.00^{*}$ \\
\hline xARM & 0.50 & xARM & $0.04^{*}$ & $\mathrm{~Pb}$ & 0.20 & $\mathrm{~Pb}$ & 0.30 \\
\hline \multirow[t]{7}{*}{ SIRM } & 0.30 & SIRM & 0.29 & Th & 0.32 & Th & 0.17 \\
\hline & & & & $\mathrm{U}$ & 0.31 & $\mathrm{U}$ & 0.30 \\
\hline & & & & Xlf & 0.22 & Xlf & 0.23 \\
\hline & & & & Xfd & 0.50 & Xfd & 0.26 \\
\hline & & & & ARM & 0.50 & ARM & 0.09 \\
\hline & & & & xARM & 0.50 & xARM & 0.09 \\
\hline & & & & SIRM & 0.50 & SIRM & 0.49 \\
\hline
\end{tabular}

${ }^{*}$ Statistically significant at $\mathrm{p}<0.05$ and suggesting a non-normal distribution.

Consistent with an existing procedure for selecting statistically robust composite signatures for discriminating potential sediment sources (Collins et al. (2012a), all the conservative fingerprint properties were tested for discriminatory efficiency using three different statistical tests: KruskalWallis H-Test (KW-H), Principal Component Analysis (PCA) and genetic algorithm discriminant function analysis (GA-DFA), to select the composite signature consisting of $n+1$ fingerprints (where $\mathrm{n}$ is the number of sources) offering the best discrimination of the potential catchment sediment sources. The rationale was that the use of three independent statistical procedures to select different sets of composite fingerprints will increase the robustness of the source discrimination and the corresponding mass balance modelling. The KW-H test is a nonparametric version of the classic oneway ANOVA (Gibbons, 2011; Hollander and Wolfe, 2013). It was applied to examine the ability of individual fingerprints to distinguish the catchment source samples. The majority of the individual tracers showed statistically significant differences between surface and subsurface sources (Table 2). The PCA provides a useful means to analyse variance in tracer datasets and reduces dimensionality by using an orthogonal transformation to convert observations of potentially correlated variables into uncorrelated principal components (Jolliffe, 2002). The loadings and variance explained by the first two principal components for each sediment sample are shown in Table 3. The Chi-square and pvalues associated with each individual property passing the Kruskal-Wallis H-test or the PCA were ranked and the top 3 selected as the optimum composite fingerprints. Each optimum composite 
fingerprint was run through a step-wise DFA to calculate the percentage of correct source category sample classification accorded by each individual fingerprint property within, and the overall, composite fingerprint. The DFA also accorded the opportunity to calculate a tracer discriminatory weighting (TDW) (Collins et al., 2012a) for the mass balance modelling. The use of this weighting has been shown to help constrain the uncertainty ranges associated with source proportions (Collins $e t$ al., 2010b; Wilkinson et al., 2013). 
Table 2. Kruskal-Wallis H-test results for selecting fingerprint properties for distinguishing catchment surface and subsurface sediment sources

\begin{tabular}{|c|c|c|c|c|c|c|c|c|c|c|c|c|c|c|}
\hline \multicolumn{6}{|l|}{ Site 1} & \multicolumn{9}{|l|}{ Site 2} \\
\hline \multicolumn{3}{|c|}{$2000-2010$} & \multicolumn{3}{|c|}{$1958-2000$} & \multicolumn{3}{|c|}{ 2000-2010 } & \multicolumn{3}{|c|}{$1958-2000$} & \multicolumn{3}{|c|}{ Pre-1958 } \\
\hline Property & H-value & $\mathrm{p}$-value & Property & H-value & p-value & Property & H-value & p-value & Property & H-value & $p$-value & Property & H-value & p-value \\
\hline $\mathrm{Mn}$ & 9.375 & $0.002^{*}$ & $\mathrm{Mn}$ & 9.375 & $0.002^{*}$ & $\mathrm{Mn}$ & 10.249 & $0.001^{*}$ & $\mathrm{Mn}$ & 10.249 & $0.001^{*}$ & $\mathrm{Mn}$ & 10.249 & $0.001^{*}$ \\
\hline $\mathrm{Ni}$ & 9.375 & $0.002^{*}$ & $\mathrm{Ni}$ & 9.375 & $0.002^{*}$ & $\operatorname{Pr}$ & 9.880 & 0.373 & $\mathrm{Sc}$ & 9.422 & $0.002^{*}$ & $\mathrm{Sc}$ & 9.422 & $0.002^{*}$ \\
\hline $\mathrm{Na}$ & 8.513 & $0.004^{*}$ & $\mathrm{Na}$ & 8.513 & $0.004^{*}$ & $\mathrm{Sc}$ & 9.422 & $0.002^{*}$ & $\mathrm{Y}$ & 6.127 & $0.013^{*}$ & $\mathrm{Y}$ & 6.127 & $0.013^{*}$ \\
\hline B & 8.513 & $0.004^{*}$ & B & 8.513 & $0.004^{*}$ & $\mathrm{Ce}$ & 7.593 & 0.817 & Co & 3.664 & 0.056 & Co & 3.664 & 0.056 \\
\hline Co & 8.098 & $0.004^{*}$ & Co & 8.098 & $0.004^{*}$ & $\mathrm{Cd}$ & 6.127 & $0.013^{*}$ & $\mathrm{Xfd}$ & 2.109 & 0.146 & $\operatorname{Pr}$ & 2.109 & 0.146 \\
\hline $\mathrm{Cu}$ & 8.098 & $0.004^{*}$ & $\mathrm{Cu}$ & 8.098 & $0.004^{*}$ & $\mathrm{Y}$ & 5.808 & $0.016^{*}$ & $\mathrm{Nd}$ & 2.109 & 0.146 & $\mathrm{Sr}$ & 1.657 & 0.198 \\
\hline $\mathrm{Ba}$ & 5.825 & $0.016^{*}$ & $\mathrm{Ba}$ & 5.825 & $0.016^{*}$ & $\mathrm{Nd}$ & 4.746 & $0.029^{*}$ & $\mathrm{Sr}$ & 1.657 & 0.198 & $\mathrm{Ba}$ & 1.259 & 0.262 \\
\hline $\mathrm{Ce}$ & 5.825 & $0.016^{*}$ & $\mathrm{Ce}$ & 5.825 & $0.016^{*}$ & $\mathrm{Co}$ & 3.664 & 0.056 & $\mathrm{Ba}$ & 1.259 & 0.262 & $\mathrm{U}$ & 0.794 & 0.373 \\
\hline $\mathrm{Cd}$ & 5.315 & $0.021^{*}$ & $\mathrm{Sr}$ & 5.315 & $0.021^{*}$ & $\mathrm{Au}$ & 2.109 & 0.146 & $\mathrm{Ce}$ & 0.794 & 0.373 & $\mathrm{Ce}$ & 0.794 & 0.373 \\
\hline $\mathrm{Sr}$ & 4.991 & $0.025^{*}$ & $\mathrm{Ga}$ & 4.365 & $0.037^{*}$ & $\mathrm{Sr}$ & 1.657 & 0.198 & $\mathrm{Na}$ & 0.736 & 0.391 & $\mathrm{La}$ & 0.053 & 0.817 \\
\hline $\mathrm{Ga}$ & 4.365 & $0.037^{*}$ & $\mathrm{Rb}$ & 3.115 & 0.078 & $\mathrm{Ba}$ & 1.259 & 0.262 & $\mathrm{U}$ & 0.480 & 0.488 & & & \\
\hline $\mathrm{Rb}$ & 3.115 & 0.078 & $\mathrm{Tl}$ & 0.047 & 0.829 & $\mathrm{Na}$ & 0.736 & 0.391 & $\operatorname{Pr}$ & 0.070 & 0.792 & & & \\
\hline \multirow[t]{5}{*}{$\mathrm{Tl}$} & 0.047 & 0.829 & & & & $\mathrm{U}$ & 0.480 & 0.488 & $\mathrm{La}$ & 0.053 & 0.817 & & & \\
\hline & & & & & & $\mathrm{La}$ & 0.053 & 0.817 & & & & & & \\
\hline & & & & & & Xfd & 0.053 & 0.817 & & & & & & \\
\hline & & & & & & ARM & 0.053 & 0.817 & & & & & & \\
\hline & & & & & & Xarm & 0.053 & 0.817 & & & & & & \\
\hline
\end{tabular}

${ }^{*}=$ statistically significant at $\mathrm{p}<0.05$ 
Table 3. Ranked fingerprint property loadings generated using PCA for floodplain Site 1 and Site 2.

Site 1

\section{0-2010}

\begin{tabular}{llll}
\hline Property & $\mathrm{PC}^{\mathrm{a}}$ & Property & $\mathrm{PC}^{\mathrm{b}}$ \\
\hline $\mathrm{Na}$ & 0.992 & $\mathrm{Mn}$ & 0.982
\end{tabular}

$\mathrm{Mn} \quad 0.126 \mathrm{Ba} \quad 0.132$

$\begin{array}{lll}\mathrm{Ba} & 0.028 \mathrm{Na}\end{array}$

$\begin{array}{lll}\mathrm{Sr} & 0.009 \mathrm{Rb}\end{array}$

B $\quad 0.004$ Co

$\mathrm{Rb} \quad 0.002 \mathrm{Ni}$

$\begin{array}{lll}\mathrm{Co} & 0.002 \mathrm{Cu}\end{array}$

$\mathrm{Cu} \quad 0.001 \mathrm{Ce}$

$\mathrm{Ce} \quad 0.001 \mathrm{Ga}$

$\mathrm{Ga} \quad 0.001 \mathrm{Sr}$

$\mathrm{Ni} \quad 0.000 \quad \mathrm{~B}$

$\mathrm{VE} \%$

0.129

0.017

0.011

0.011

0.008

0.007

0.006

0.002

16.67
Site 2

2000-2010

$\begin{array}{llll}\text { Property } & \mathrm{PC}^{\mathrm{a}} & \text { Property } & \mathrm{PC}^{\mathrm{b}} \\ \mathrm{Na} & 0.991 & \mathrm{Mn} & 0.956\end{array}$

Mn

$0.130 \mathrm{Xfd}$

0.231

$\mathrm{Ba}$

$0.029 \mathrm{Ba}$

0.134

$0.017 \quad \mathrm{Xfd}$

$0.026 \mathrm{Na}$

0.123

$\mathrm{Sr}$

0.009 Co

0.016

Co

$0.002 \mathrm{Ce}$

0.008

$\mathrm{La}$

$0.002 \mathrm{Sr}$

0.005

$\mathrm{Ce}$

$0.001 \mathrm{Y}$

0.004

$\mathrm{Nd}$

$0.001 \mathrm{Nd}$

0.004

$0.000 \mathrm{Sc}$

0.002

$\operatorname{Pr}$

0.000 xARM

0.001

$\mathrm{Au}$

$0.000 \mathrm{U}$

0.001

$\mathrm{xARM}$

$0.000 \quad \mathrm{La}$

0.000

$\mathrm{VE} \%$

78.22 VE\%

16.82

\section{8-2000}

\begin{tabular}{|c|c|c|c|c|c|c|c|}
\hline \multicolumn{4}{|c|}{$1958-2000$} & \multicolumn{4}{|c|}{$1958-2000$} \\
\hline $\mathrm{Na}$ & 0.992 & $\mathrm{Mn}$ & 0.982 & $\mathrm{Na}$ & 0.991 & $\mathrm{Mn}$ & 0.956 \\
\hline $\mathrm{Mn}$ & 0.126 & $\mathrm{Ba}$ & 0.132 & $\mathrm{Mn}$ & 0.130 & Xfd & 0.231 \\
\hline $\mathrm{Ba}$ & 0.028 & $\mathrm{Na}$ & 0.129 & $\mathrm{Ba}$ & 0.029 & $\mathrm{Ba}$ & 0.134 \\
\hline $\mathrm{Sr}$ & 0.009 & $\mathrm{Rb}$ & 0.017 & Xfd & 0.026 & $\mathrm{Na}$ & 0.123 \\
\hline B & 0.004 & Co & 0.017 & $\mathrm{Sr}$ & 0.009 & Co & 0.016 \\
\hline $\mathrm{Rb}$ & 0.002 & $\mathrm{Ni}$ & 0.011 & $\mathrm{Co}$ & 0.002 & $\mathrm{Ce}$ & 0.008 \\
\hline Co & 0.002 & $\mathrm{Cu}$ & 0.011 & $\mathrm{La}$ & 0.002 & $\mathrm{Sr}$ & 0.005 \\
\hline $\mathrm{Cu}$ & 0.001 & $\mathrm{Ce}$ & 0.008 & $\mathrm{Ce}$ & 0.001 & $\mathrm{Y}$ & 0.004 \\
\hline $\mathrm{Ce}$ & 0.001 & $\mathrm{Ga}$ & 0.007 & $\mathrm{Nd}$ & 0.001 & $\mathrm{Nd}$ & 0.004 \\
\hline $\mathrm{Ga}$ & 0.001 & $\mathrm{Sr}$ & 0.006 & $\mathrm{Y}$ & 0.000 & $\mathrm{Sc}$ & 0.002 \\
\hline $\mathrm{Ni}$ & 0.000 & $\mathrm{~B}$ & 0.002 & $\operatorname{Pr}$ & 0.000 & $\mathrm{U}$ & 0.001 \\
\hline \multirow[t]{10}{*}{ VE\% } & 81.26 & $\mathrm{VE} \%$ & 16.67 & $\mathrm{VE} \%$ & 78.22 & VE\% & 16.82 \\
\hline & & & & \multicolumn{4}{|c|}{ Pre-1958 } \\
\hline & & & & $\mathrm{Mn}$ & 0.990 & $\mathrm{Ba}$ & 0.988 \\
\hline & & & & $\mathrm{Ba}$ & 0.142 & $\mathrm{Mn}$ & 0.142 \\
\hline & & & & $\mathrm{Co}$ & 0.017 & $\mathrm{Ce}$ & 0.041 \\
\hline & & & & $\mathrm{Sr}$ & 0.011 & $\mathrm{Sr}$ & 0.039 \\
\hline & & & & $\mathrm{Ce}$ & 0.007 & $\mathrm{La}$ & 0.028 \\
\hline & & & & $\mathrm{Y}$ & 0.004 & $\mathrm{Y}$ & 0.011 \\
\hline & & & & $\mathrm{Sc}$ & 0.002 & $\operatorname{Pr}$ & 0.007 \\
\hline & & & & $\mathrm{La}$ & 0.001 & $\mathrm{Co}$ & 0.006 \\
\hline
\end{tabular}




\begin{tabular}{llll}
$\mathrm{U}$ & 0.000 & $\mathrm{U}$ & 0.001 \\
$\mathrm{Pr}$ & 0.000 & $\mathrm{Sc}$ & 0.001 \\
$\mathrm{VE} \%$ & 90.29 & $\mathrm{VE} \%$ & 9.09 \\
\hline \multicolumn{4}{c}{} \\
& \multicolumn{3}{l}{ VE\% variance explained } \\
& a Principal component 1 \\
& ' Principal component 2
\end{tabular}

A third set of alternative composite fingerprints was obtained by applying the GA-DFA. This is a discriminant function analysis based fitness function for optimum composite signature selection using genetic algorithm optimization (Jarvis and Goodacre, 2005). The GA-DFA was iterated for each sample group using minimisation of Wilks' lambda, a stepwise selection algorithm (cf. Collins et al., 1997a) and a probability value for parameter entry of 0.05 . The GA-DFA was run three times to obtain composite fingerprints consisting of three, four and five individual properties for investigating the potential sensitivity of the source apportionment estimates to the number of tracers constituting each composite signature. Similar to the KW-H and PCA, the percentage correct classification and tracer discriminatory weightings of each composite signature were calculated.

\subsection{Sediment source apportionment using a mass balance un-mixing model}

Sediment sources apportionment was completed using a numerical mass balance un-mixing model first described by Collins et al. (1997a). This model has been used in many studies (Bottrill et al., 2000; Collins and Walling, 2007; Gruszowski et al., 2003; Minella et al., 2008; Russell et al., 2001; Walling et al., 2008) and was recently revised by Collins et al. (2010a, 2012ab). The model operates through minimising the sum of squares of the weighted relative errors in the objective function by changing the relative source proportions:

$$
\sum_{i=1}^{n}\left\{\left(C_{i}-\left(\sum_{s=1}^{m} P_{s} S_{s i} Z_{s}\right)\right) / C_{i}\right\}^{2} W_{i} \quad \text { Eq.1 }
$$


where: $\mathrm{Ci}=$ deviate median concentration of fingerprint property (i) in each floodplain sediment core sample; Ps = the optimised percentage contribution from each sediment source category (s); Ssi = deviate median concentration of fingerprint property (i) in source category (s); Z= particle size correction factor for source category $(\mathrm{s})$; Wi = tracer discriminatory weighting; $\mathrm{n}=$ number of fingerprint properties comprising the optimum composite fingerprint; $\mathrm{m}=$ number of sediment source categories. The model solutions were subject to two boundary constraints:

$$
\begin{array}{ll}
0 \leq P_{s} \leq 1 & \text { Eq. } 2 \\
\sum_{s=1}^{n} P_{s}=1 & \text { Eq. } 3
\end{array}
$$

Many mass balance model formulations include particle size content correction factors (Collins et al., 2013; Haddadchi et al., 2014; Russell et al., 2001; Walling et al., 2008) to take account of the effects of contrasts in grain-size composition between sediment and source material samples on fingerprint property values. Consistent with other related studies (Fox and Papanicolaou, 2008; Walling et al., 1999), the particle size correction factor was calculated using a ratio of the mean specific surface area $\left(\mathrm{m}^{2} \mathrm{~g}-1\right)$ of the floodplain sediment samples to the corresponding mean values for each potential source. The tracer discriminatory power weighting was based on the relative outputs of each individual fingerprint property comprising the specific composite signature identified using the statistical tests following Collins et al. (2010a). Thus the discriminatory power of the property providing the lowest discrimination (\%) of the sediment source samples was assigned a value of 1.0 and the corresponding weightings for the rest of the properties were calculated using the ratio of their discriminatory power to that of the weakest property in any specific composite signature. Given the similarity between the estimated values of the tracer discriminatory weighting, no standardisation of these values was required.

The mass balance model was operated using a Monte Carlo framework. The input values to the model were obtained from fingerprint property distributions generated for both the source and floodplain sediment samples using the median as a robust estimator of location and the scaler Qn (Collins et al., 2012a). The mixing model was repeatedly solved either 5000 or 30000 times for each composite fingerprint and each individual sediment sample comprising the floodplain cores, to assess whether the number of model iterations affects the source apportionment results and associated goodness-of-fit (GOF). The model iterations were undertaken on the basis of a stratified approach based on Latin Hypercube Sampling (McKay et al., 1979; Collins et al., 2012a). A local, as opposed to global 
optimization algorithm, was used to identify solutions to the mass balance model. Research has suggested that local optimization algorithms can be less efficient as they fail to identify globally representative solutions. But in a case like the one presented here where there are only two potential sediment sources and each specific composite signature consists of only a few individual fingerprint properties ( $n=3,4$ or 5 ), the local optimization search tool performs better than the GA-driven equivalent. Outputs from the Monte Carlo analysis were summarized as output probability density functions (pdfs).The pdfs were used to estimate relative frequency-weighted average median contributions from the individual sediment sources (cf. Collins et al., 2012a) and to estimate uncertainty ranges in the predicted source proportions. Two GOF estimators were compared to assess the robustness of the optimised mixing model solutions:

$$
\begin{aligned}
& \mathrm{GOF}=1-\left[\frac{1}{\mathrm{n}} * \sum_{\mathrm{i}=1}^{\mathrm{n}}\left\{\left(\mathrm{C}_{\mathrm{i}}-\left(\sum_{\mathrm{s}=1}^{\mathrm{m}} \mathrm{P}_{\mathrm{s}} \mathrm{S}_{\mathrm{si}}\right)\right) / \mathrm{C}_{\mathrm{i}}\right\}^{2} \mathrm{~W}_{\mathrm{i}}\right] \\
& \mathrm{GOF}=\left(1-\left[\sqrt{\sum_{\mathrm{i}=1}^{\mathrm{n}}}\left\{\left(\mathrm{C}_{\mathrm{i}}-\left(\sum_{\mathrm{s}=1}^{\mathrm{m}} \mathrm{P}_{\mathrm{s}} \mathrm{S}_{\mathrm{si}}\right)\right) / \mathrm{C}_{\mathrm{i}}\right\}^{2} \mathrm{~W}_{\mathrm{i}}\right]\right) / \mathrm{n} \quad \text { Eq.5 }
\end{aligned}
$$

\section{Results and discussion}

\subsection{Tracer conservation and statistical source discrimination}

The tracer range test (Table 4) shows that both geochemical and mineral-magnetic properties failed the test in each period. There were more non-conservative properties in the recent sediment than in the older sediment. The opposite was expected as post-depositional tracer transformation normally affects historical sediment more than the recent sediment (Belmont et al., 2014; D'Haen et al., 2012). Thus, to accommodate these conservation results any properties failing the range test in the upper part of the core (recent sediment) were not considered for the rest of the core since the anomaly of more properties being non-conservative in the upper sections could be an indication of post-depositional fingerprint property transformation over time. Proceeding with sediment source modelling using fingerprint properties that potentially violate the mass conservation assumption would increase the modelling output uncertainty (Belmont et al., 2014; Collins et al., 2010a; D’Haen et al., 2012; Koiter et al., 2013; 
Krause et al., 2003; Pulley et al., 2015; Sherrif et al., 2015; Smith and Blake, 2014). It is important to bear in mind that the range test confirms the absence of major property transformation rather than the occurrence of any transformation. 
Table 4. The sediment tracer properties which failed the mass conservativeness test. The results for the uppermost sediment were used to screen properties for the two lower sections.

\begin{tabular}{|c|c|c|c|c|}
\hline \multicolumn{2}{|l|}{ Site 1} & \multicolumn{3}{|l|}{ Site 2} \\
\hline $2000-2010$ & $1958-2000$ & $2000-2010$ & $1958-2000$ & Pre-1958 \\
\hline $\mathrm{Li}$ & $\mathrm{Mg}$ & $\mathrm{Li}$ & B & B \\
\hline $\mathrm{Mg}$ & $\mathrm{Ca}$ & B & $\mathrm{Mg}$ & $\mathrm{Na}$ \\
\hline $\mathrm{Al}$ & $\mathrm{Sc}$ & $\mathrm{Mg}$ & $\mathrm{Al}$ & $\mathrm{Mg}$ \\
\hline K & $\mathrm{Fe}$ & $\mathrm{Al}$ & $\mathrm{K}$ & $\mathrm{Ca}$ \\
\hline $\mathrm{Ca}$ & $\mathrm{Cd}$ & K & $\mathrm{Ca}$ & $\mathrm{Ru}$ \\
\hline $\mathrm{Sc}$ & $\mathrm{La}$ & $\mathrm{Ca}$ & $\mathrm{Ru}$ & $\mathrm{Rh}$ \\
\hline V & $\operatorname{Pr}$ & V & $\mathrm{Rh}$ & $\mathrm{Pd}$ \\
\hline $\mathrm{Cr}$ & $\mathrm{Nd}$ & $\mathrm{Cr}$ & $\mathrm{Pd}$ & $\mathrm{Nd}$ \\
\hline $\mathrm{Fe}$ & $\mathrm{Sm}$ & $\mathrm{Fe}$ & $\mathrm{Cd}$ & $\mathrm{Sm}$ \\
\hline $\mathrm{Zn}$ & $\mathrm{Eu}$ & $\mathrm{Ni}$ & $\mathrm{Eu}$ & $\mathrm{Yb}$ \\
\hline As & Gd & $\mathrm{Cu}$ & $\mathrm{Gd}$ & $\mathrm{Lu}$ \\
\hline Y & $\mathrm{Tb}$ & $\mathrm{Zn}$ & $\mathrm{Tb}$ & $\operatorname{Re}$ \\
\hline $\mathrm{Pd}$ & Dy & $\mathrm{Ga}$ & Dy & Os \\
\hline Cs & Но & As & Ho & Ir \\
\hline $\mathrm{La}$ & $\mathrm{Er}$ & $\mathrm{Rb}$ & $\mathrm{Er}$ & $\mathrm{Pt}$ \\
\hline $\operatorname{Pr}$ & $\mathrm{Tm}$ & $\mathrm{Ru}$ & $\mathrm{Tm}$ & Xlf \\
\hline $\mathrm{Nd}$ & $\mathrm{Yb}$ & $\mathrm{Rh}$ & $\mathrm{Lu}$ & Xfd \\
\hline $\mathrm{Sm}$ & $\mathrm{Lu}$ & $\mathrm{Pd}$ & $\mathrm{Re}$ & ARM \\
\hline $\mathrm{Eu}$ & Th & $\mathrm{Cs}$ & Os & Xarm \\
\hline Gd & & $\mathrm{Sm}$ & Ir & SIRM \\
\hline $\mathrm{Tb}$ & & $\mathrm{Eu}$ & $\mathrm{Pt}$ & \\
\hline Dy & & Gd & $\mathrm{Au}$ & \\
\hline Ho & & $\mathrm{Tb}$ & $\mathrm{Pb}$ & \\
\hline $\mathrm{Er}$ & & Dy & Xlf & \\
\hline $\mathrm{Tm}$ & & Ho & SIRM & \\
\hline $\mathrm{Yb}$ & & $\mathrm{Er}$ & & \\
\hline $\mathrm{Lu}$ & & $\mathrm{Tm}$ & & \\
\hline Os & & $\mathrm{Yb}$ & & \\
\hline $\mathrm{Pb}$ & & $\mathrm{Lu}$ & & \\
\hline Th & & $\operatorname{Re}$ & & \\
\hline $\mathrm{U}$ & & Os & & \\
\hline Xlf & & Ir & & \\
\hline Xfd & & $\mathrm{Pt}$ & & \\
\hline ARM & & $\mathrm{Tl}$ & & \\
\hline xARM & & $\mathrm{Pb}$ & & \\
\hline SIRM & & Th & & \\
\hline & & $\begin{array}{l}\text { XIf } \\
\text { SIRM }\end{array}$ & & \\
\hline
\end{tabular}


All the conservative fingerprint properties were used in the statistical sediment source discrimination using the KW-H, PCA and GA-DFA to select three different optimum composite fingerprints. Table 5 and Table 6 show the optimum composite fingerprints selected by KW-H and PCA for discriminating between surface and subsurface sediment sources. Similar individual sediment properties (Mn, Ni, and $\mathrm{Na}$, and $\mathrm{Mn}$ and $\mathrm{Sc}$ ) were consistently selected in the first two and all the three sections of the sediment core in Site 1 and Site 2, respectively. Also $\mathrm{Na}$ and $\mathrm{Mn}$ were selected by KW-H and PCA for the first two sediment sections in both sites. There was consistency in most of the individual fingerprints selected by the KW-H and PCA statistical procedures for the two floodplain sites. The GA-DFA results are presented in Table 7. As opposed to KW-H and PCA whereby there was consistency in the individual properties selected in the composite signatures for the two sites, each composite signature selected by GA-DFA generally consisted of a different set of properties reflecting the basis of the GA approach driving this specific test.

The optimum signatures selected using KW-H classified correctly $72.6 \%-74.2 \%$ of the most recent sediment samples in both sites, $72.6 \%-74.2 \%$ of the $1958-2000$ sediment and $74.2 \%$ of the pre-1958 sediment. The percentage correct discrimination of the sediment sources accorded by the composite signatures were constant for both sediment sections at Site 1 and for the three sections at Site 2 . The optimum composite fingerprint selected by PCA classified correctly $74.2 \%-78.9 \%$ of the most recent sediment samples, $74.2 \%-78.9 \%$ of the $1958-2000$ sediment and $79.9 \%$ of the pre-1958 sediment. The GA-DFA results presented an opportunity to assess whether an increase in the number of individual fingerprints in each composite signature (from 3 up to 4 or 5) affects the percentage correct classification of the catchment source samples or the un-mixing model GOF. For illustrative purposes and ease of comparison, only the composite fingerprint with the highest percentage correct discrimination of the two catchment sediment sources selected by each GA-DFA run (i.e. using 3, 4 or 5 properties in each signature) is presented in Table 7. It can be observed that the percentage of catchment source samples classified correctly by the GA-DFA composite signatures did not improve by increasing the fingerprint properties in each composite signature for both sites. Inclusion of high numbers of individual tracers in composite signatures can improve signature discriminatory power but clearly this must be considered in the context of the overall need to minimise un-mixing model errors. This point is discussed further in a subsequent section. Most of the optimum composite fingerprints were unable to achieve the minimum threshold of $85 \%$ correct discrimination used by most sediment source fingerprinting studies (Collins et al. 1997a; Collins et al., 2010a; Owens et al., 1999; Stone et al., 2014; Walling et al., 2008). Consequently, a higher degree of uncertainty is associated with the estimates of sediment source apportionment given the weaker source discrimination using the datasets collected here. 
Table 5. Optimum composite fingerprints selected by the KW-H test for discriminating between surface and subsurface sediment sources

Site 1

2000-2010

\begin{tabular}{lllll}
\hline Property & $\begin{array}{l}\text { Chi- } \\
\text { square }\end{array}$ & p-Value & $\%^{\mathrm{a}}$ & TDW $^{\mathrm{b}}$ \\
\hline $\mathrm{Mn}$ & 9.375 & 0.002 & 72.6 & 1.00 \\
$\mathrm{Ni}$ & 9.375 & 0.002 & 59.7 & 1.10 \\
$\mathrm{Na}$ & 8.513 & 0.004 & 65.8 & 1.06 \\
Total $^{\mathrm{c}}$ & & & 72.6 &
\end{tabular}

1958-2000

\begin{tabular}{lllll}
\hline $\mathrm{Mn}$ & 9.375 & 0.002 & 72.6 & 1.22 \\
$\mathrm{Ni}$ & 9.375 & 0.002 & 59.7 & 1.00 \\
$\mathrm{Na}$ & 8.513 & 0.004 & 65.8 & 1.10 \\
Total $^{c}$ & & & 72.6 &
\end{tabular}

a \% source samples classified correctly by individual tracers

${ }^{\mathrm{b}}$ tracer discriminatory weighting used in the un-mixing modelling

c $\%$ source samples classified correctly by composite signature
Site 2

2000-2010

\begin{tabular}{lllll}
\hline Property & $\begin{array}{l}\text { Chi- } \\
\text { square }\end{array}$ & p-Value & $\%^{\mathrm{a}}$ & $\mathrm{TDW}^{\mathrm{b}}$ \\
\hline $\mathrm{Mn}$ & 10.249 & 0.001 & 74.2 & 1.30 \\
$\mathrm{Cd}$ & 6.127 & 0.013 & 71.4 & 1.25 \\
$\mathrm{Sc}$ & 9.422 & 0.002 & 57.2 & 1.00 \\
Total $^{c}$ & & & 74.2 &
\end{tabular}

\begin{tabular}{lllll}
\multicolumn{1}{l}{ 1958-2000 } & & & \\
\hline $\mathrm{Mn}$ & 10.249 & 0.001 & 74.2 & 1.30 \\
$\mathrm{Sc}$ & 9.422 & 0.002 & 57.2 & 1.00 \\
$\mathrm{Y}$ & 6.127 & 0.013 & 62.6 & 1.09 \\
Total $^{\mathrm{c}}$ & & & 74.2 &
\end{tabular}

\begin{tabular}{lllll}
\multicolumn{2}{l}{ Pre-1958 } & & & \\
\\
$\mathrm{Mn}$ & 10.249 & 0.001 & 74.2 & 1.30 \\
$\mathrm{Sc}$ & 9.422 & 0.002 & 57.2 & 1.00 \\
$\mathrm{Y}$ & 6.127 & 0.013 & 62.6 & 1.09 \\
Total $^{\mathrm{c}}$ & & & 74.2 &
\end{tabular}


Table 6. Optimum composite fingerprints selected by PCA for discriminating between surface and subsurface sediment sources.

Site 1

2000-2010

\begin{tabular}{lll}
\hline Property & $\%^{\mathrm{a}}$ & $\mathrm{TDW}^{\mathrm{b}}$ \\
\hline $\mathrm{Na}$ & 65.8 & 1.00 \\
$\mathrm{Mn}$ & 72.6 & 1.10 \\
$\mathrm{Ba}$ & 69.5 & 1.06 \\
Total $^{\mathrm{c}}$ & 78.9 &
\end{tabular}

1958-2000

$\mathrm{Na}$

$\mathrm{Mn}$

$\mathrm{Ba}$

Total $^{c}$

\begin{tabular}{lll} 
1958-2000 & & \\
\hline $\mathrm{Na}$ & 65.8 & 1.00 \\
$\mathrm{Mn}$ & 72.6 & 1.10 \\
$\mathrm{Ba}$ & 65.8 & 1.06 \\
Total $^{\mathrm{c}}$ & 78.9 &
\end{tabular}

Site 2

2000-2010

\begin{tabular}{lll}
\hline Property & $\mathbf{\%}^{\mathrm{a}}$ & $\mathrm{TDW}^{\mathrm{b}}$ \\
\hline $\mathrm{Na}$ & 67.8 & 1.32 \\
$\mathrm{Mn}$ & 74.2 & 1.45 \\
Xfd & 51.3 & 1.00 \\
Total $^{\mathrm{c}}$ & 74.2 &
\end{tabular}

\begin{tabular}{lll} 
1958-2000 & & \\
\hline $\mathrm{Na}$ & 67.8 & 1.32 \\
$\mathrm{Mn}$ & 74.2 & 1.45 \\
$\mathrm{Xfd}$ & 51.3 & 1.00 \\
Total $^{\mathrm{c}}$ & 74.2 & \\
& & \\
Pre-1958 & & \\
$\mathrm{Mn}$ & 74.2 & 1.04 \\
$\mathrm{Ba}$ & 71.2 & 1.00 \\
$\mathrm{Co}$ & 74.2 & 1.04 \\
Total $^{\mathrm{c}}$ & 79.9 &
\end{tabular}

\footnotetext{
a \% source samples classified correctly by individual tracers

${ }^{\mathrm{b}}$ tracer discriminatory weighting used in the un-mixing modelling

c $\%$ source samples classified correctly by composite signature
} 
Table 7. Optimum composite fingerprints selected by the GA-DFA for discriminating between surface and subsurface sediment sources

Site 1

2000-2010

Run $1^{\mathrm{a}}$

\begin{tabular}{lrr}
\hline Property & \multicolumn{1}{c}{${ }^{\mathrm{d}}$} & $\mathrm{TDW}^{\mathrm{e}}$ \\
$\mathrm{Tl}$ & 73 & 1.05 \\
$\mathrm{Cu}$ & 75.9 & 1.09 \\
$\mathrm{Ba}$ & 69.5 & 1 \\
Total $^{\mathrm{f}}$ & 84.9 &
\end{tabular}

\begin{tabular}{llr} 
Run $2^{\mathrm{b}}$ & & \\
\hline Property & $\%^{\mathrm{d}}$ & TDW $^{\mathrm{e}}$ \\
Cd & 69.7 & 1 \\
Co & 72.6 & 1.04 \\
$\mathrm{Ba}$ & 69.5 & 1.09 \\
B & 66.5 & 1.05 \\
Total $^{\mathrm{f}}$ & 84.7 &
\end{tabular}

Run $3^{\mathrm{c}}$

Property $\%{ }^{\mathrm{d}} \quad \mathrm{TDW}^{\mathrm{e}}$

Cd

$69.7 \quad 1.11$

$\mathrm{Cu}$

$75.9 \quad 1.11$

$\mathrm{Na}$

$65.8 \quad 1.15$

$73 \quad 1.06$

$\mathrm{G}^{\mathrm{a}} \quad 72.8 \quad 1$

Total $^{\mathrm{f}} \quad 84.9$

1958-2000

\begin{tabular}{lrrlrrlrr}
\hline $\mathrm{Tl}$ & 73 & 1.05 & $\mathrm{Sr}$ & 66.2 & 1 & $\mathrm{Cu}$ & 75.9 & 1.15 \\
$\mathrm{Cu}$ & 75.9 & 1.09 & $\mathrm{Co}$ & 72.6 & 1.1 & $\mathrm{Rb}$ & 69.7 & 1.05 \\
$\mathrm{Ba}$ & 65.8 & 1 & $\mathrm{~B}$ & 66.5 & 1.01 & $\mathrm{Sr}$ & 66.2 & 1 \\
Total $^{\mathrm{f}}$ & 84.9 & & $\mathrm{Ba}$ & 65.8 & 1.05 & $\mathrm{Ga}$ & 72.8 & 1.1 \\
& & & Total $^{\mathrm{f}}$ & 78.9 & & $\mathrm{Co}$ & 72.6 .0 & 1.1 \\
& & & & & & & \\
& & & & & & & & \\
\end{tabular}

Site 2

2000-2010

\begin{tabular}{lrr}
\hline $\mathrm{Mn}$ & 74.2 & 1.19 \\
$\mathrm{Y}$ & 62.6 & 1 \\
$\mathrm{Sr}$ & 68.1 & 1.09 \\
Total $^{\mathrm{f}}$ & 74.2 &
\end{tabular}

1958-2000

\begin{tabular}{lrr}
\hline $\mathrm{Ce}$ & 48.7 & 1 \\
$\mathrm{Ba}$ & 71.2 & 1.46 \\
$\mathrm{Co}$ & 74.2 & 1.52 \\
Total $^{\mathrm{f}}$ & 79.9 &
\end{tabular}

\section{Pre-1958}

\begin{tabular}{lrr}
\hline $\mathrm{Pr}$ & 54.4 & 1 \\
$\mathrm{Mn}$ & 74.2 & 1.36 \\
$\mathrm{Ba}$ & 71.2 & 1.31 \\
Total $^{\mathrm{f}}$ & 82.8 &
\end{tabular}

\begin{tabular}{|c|c|c|c|c|c|}
\hline Co & 74.2 & 1.52 & $\mathrm{Ba}$ & 71.2 & 1.46 \\
\hline $\mathrm{Na}$ & 67.8 & 1.39 & $\operatorname{Pr}$ & 54.4 & 1 \\
\hline $\mathrm{Ce}$ & 48.7 & 1 & $\mathrm{Ce}$ & 48.7 & 1.12 \\
\hline $\mathrm{Nd}$ & 54.4 & 1.12 & $\mathrm{U}$ & 62.9 & 1.29 \\
\hline \multirow[t]{2}{*}{ Total $^{\mathrm{f}}$} & 77 & & $\mathrm{Na}$ & 67.81 & 1.39 \\
\hline & & & Tota & 82.7 & \\
\hline
\end{tabular}

\begin{tabular}{lll}
\hline $\mathrm{Ba}$ & 71.2 & 1.46
\end{tabular}

\begin{tabular}{lll}
\hline $\mathrm{Ba}$ & 71.2 & 1.46
\end{tabular}

$\begin{array}{llllll}\operatorname{Pr} & 54.4 & 1.12 & \operatorname{Pr} & 54.4 & 1.12\end{array}$

$\begin{array}{llllll}\mathrm{Ce} & 48.7 & 1 & \mathrm{Ce} & 48.7 & 1\end{array}$

$\begin{array}{llllll}\mathrm{U} & 62.9 & 1.29 & \mathrm{U} & 62.9 & 1.29\end{array}$

$\begin{array}{lllll}\text { Total }^{\mathrm{f}} & 82.7 & \mathrm{Na} & 67.8 & 1.39\end{array}$

Total $^{\mathrm{f}} \quad 82.7$

\begin{tabular}{|c|c|c|c|c|c|}
\hline $\operatorname{Pr}$ & 54.4 & 1.12 & $\mathrm{Ce}$ & 48.7 & 1 \\
\hline $\mathrm{U}$ & 62.9 & 1.29 & $\operatorname{Pr}$ & 54.4 & 1.12 \\
\hline $\mathrm{Ce}$ & 48.7 & 1 & $\mathrm{Sr}$ & 68.1 & 1.4 \\
\hline $\mathrm{Ba}$ & 71.2 & 1.46 & $\mathrm{Ba}$ & 71.2 & 1.46 \\
\hline \multirow[t]{2}{*}{ Total $^{\mathrm{f}}$} & 82.7 & & $\mathrm{U}$ & 62.9 & 1.29 \\
\hline & & & Total $^{f}$ & 82.7 & \\
\hline
\end{tabular}


${ }^{\text {a }}$ GA-DFA run with for composite fingerprint with a maximum of 3 properties

${ }^{\mathrm{b}}$ GA-DFA run with for composite fingerprint with a maximum of 4 properties

${ }^{\mathrm{c}}$ GA-DFA run with for composite fingerprint with a maximum of 5 properties

$\mathrm{d} \%$ source samples classified correctly by individual tracers

${ }^{\mathrm{e}}$ tracer discriminatory weighting used in the un-mixing modelling

f\% source samples classified correctly by composite signature

\subsection{Mass balance model performance}

Many sediment source fingerprinting studies compare un-mixing model performance on the basis of estimates of the model GOF. Often the GOF estimators are based on reducing absolute or squared errors between the modelled and measured sediment properties. A commonly used objective function (Eq. 4) includes multiplication by the inverse of the number of properties in the composite signature in question. The implication of this is that an improved GOF with measured sediment tracer concentrations is achieved as the number of fingerprint properties entered into the model is increased. However, some recent work (e.g. Laceby and Olley, 2015) has underscored the risk of divergence between the widely-used GOF estimator represented by Eq. 4 and the more traditional estimator based on the absolute error (cf. Collins et al., 1997; Walling and Collins, 2000). This divergence reflects the counterintuitive performance of Eq. 4 in that a composite signature with more constituent tracers will typically return an improved GOF, whereas in the case of using the absolute error, the inclusion of additional tracers will likely degrade the GOF. In recognition of these potential issues, we used a modified GOF estimator based on the square root of the objective function - i.e. based on the absolute mixing model error, normalised by the number of tracers in any given composite signature. Figure 3 shows the relationship between the two GOF estimators. The R2 values of 0.86 and 0.87 show that the differences between the two GOF estimators is small in the case of the dataset used here. Regardless, the GOF estimator represented by Eq. 5 was adopted for inclusion in further data processing. The GOF of each composite fingerprint, using Eq. 5, for the two Monte Carlo analyses (Table 8), shows that increasing the number of individual fingerprints included in the composite signatures from 3 to 5 did not result in a better model GOF. This is contrary to findings of some studies that have reported that uncertainty in sediment source fingerprinting can be reduced by increasing the number of tracers (Martinez-Carreras et al., 2008; Poleto et al., 2009: Sherriff et al., 2015). Increasing the number of model iterations from 5000 to 30000 per floodplain core section did not improve the model efficiency in predicting the measured tracer property values of the floodplain sediment samples. In fact, most of the model GOF estimates were exactly the same for both sets of repeat iterations. These results support the use of $\leq 5000$ model iterations reported by many previous sediment source fingerprinting studies 
(Collins et al., 2010a, 2012, 2013; Hughes et al., 2009; Pulley et al., 2014; Smith and Blake, 2014; Stone et al., 2014; Wilkinson et al., 2013). The lowest GOF (average GOF of 0.79 and 0.75 for 5000 and 30000 iterations, respectively), was recorded for the upper-most sediment slice at Site 2. 

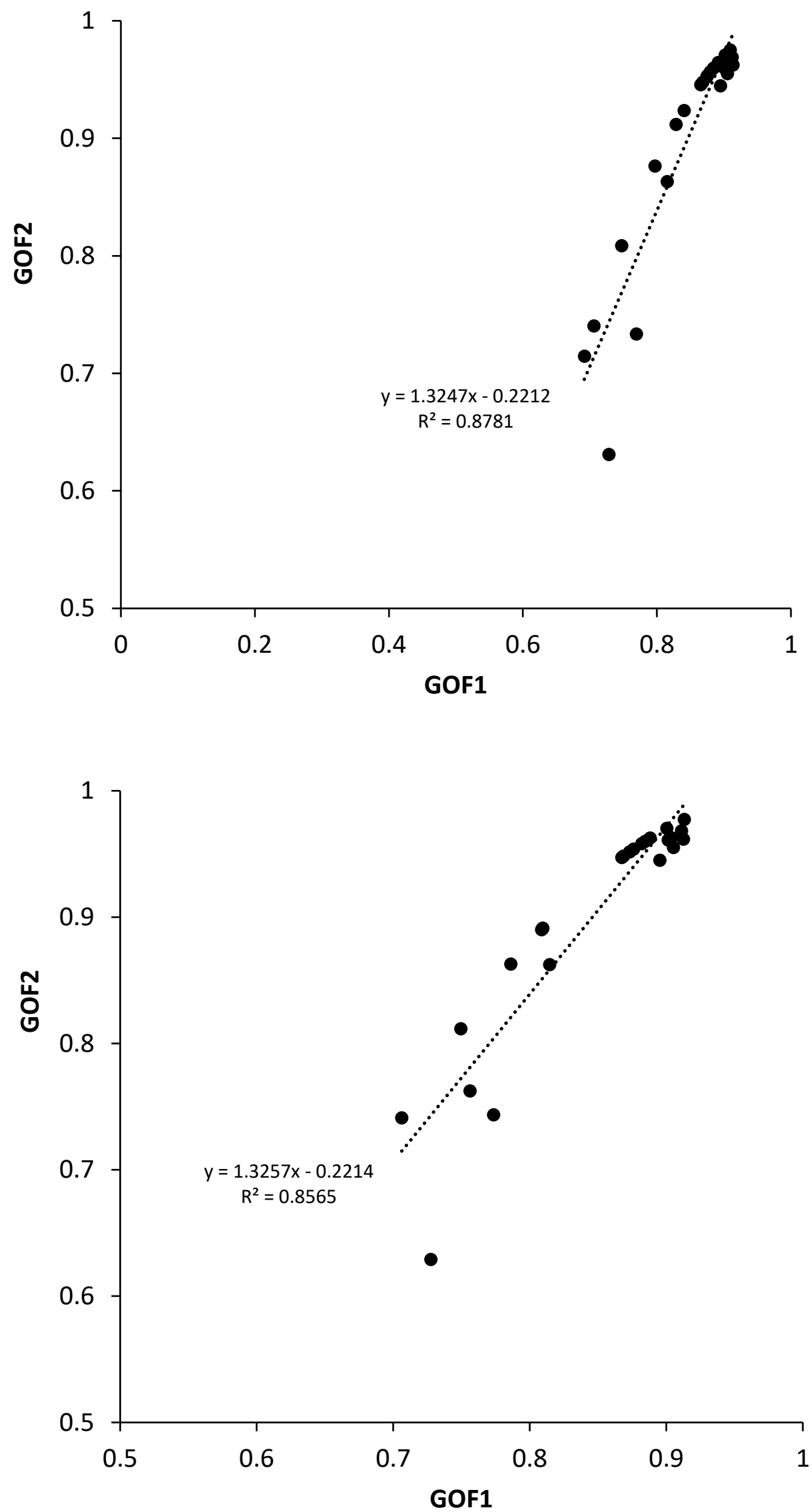

Figure 3. Plots showing the relationships between the two model GOF estimators used. 
The output probability density functions of the 5000 and 30000 iterations were plotted and used to estimate relative frequency-weighted average median contributions (Collins et al., 2012a) from the individual sediment sources and to estimate uncertainty ranges of the predicted median source proportions. We show only the pdfs for each composite fingerprint signature using 5000 iterations (Figure 4) as the pdfs are not significantly different from those obtained using 30000 iterations. The pdfs and the predicted median contributions from each source (Table 8) show that the range of feasible contributions of sediment from surface and subsurface sources was very broad (1-100\%). According to Collins et al. (2010a) the usefulness of source apportionment data generated using Monte Carlo routines depends upon how narrow the contribution ranges are for specific sources. However, in situations where large ranges exist, it is informative to take account of such ranges in estimating relative frequencyweighted average median source proportions (Collins et al., 2012a). In this case, the final estimate of the relative frequency-weighted average median source contributions was generated on the basis of a weighting (cf. Collins et al., 2014) combining the GOF and corresponding discriminatory power (\%) of each composite signature (Table 9). Only composite signatures that yielded a GOF higher than $80 \%$ were used to estimate the overall relative frequency-weighted average median source proportions. The GOF minimum of $80 \%$ has been used as a measure of reliability of mass balance model results in various fingerprinting studies (Martinez-Carreras et al., 2008; Collins et al., 2010a; Evrard et al., 2011; Mckinley et al., 2013). 
Table 8. The goodness-of-fit associated with each composite fingerprint for the model iterations in each period.

\begin{tabular}{|c|c|c|c|c|}
\hline Period & $\begin{array}{l}\text { Optimum composite } \\
\text { signature }\end{array}$ & $\begin{array}{l}\text { Number of } \\
\text { properties }\end{array}$ & $\mathrm{GOF}^{\mathrm{a}}$ & $\mathrm{GOF}^{\mathrm{b}}$ \\
\hline \multirow{6}{*}{$2000-2010$} & KW-H & 3 & 0.87 & 0.83 \\
\hline & PCA & 3 & 0.81 & 0.84 \\
\hline & GA-DFA 1 & 3 & 0.75 & 0.75 \\
\hline & GA-DFA 2 & 4 & 0.81 & 0.82 \\
\hline & GA-DFA 3 & 5 & 0.73 & 0.73 \\
\hline & Average & & 0.79 & 0.79 \\
\hline \multirow{7}{*}{$1958-2000$} & KW-H & 3 & 0.87 & 0.84 \\
\hline & PCA & 3 & 0.87 & 0.87 \\
\hline & GA-DFA 1 & 3 & 0.90 & 0.87 \\
\hline & GA-DFA 2 & 4 & 0.91 & 0.90 \\
\hline & GA-DFA 3 & 5 & 0.91 & 0.91 \\
\hline & Average & & 0.89 & 0.88 \\
\hline & KW-H & 3 & 0.89 & 0.84 \\
\hline \multirow{5}{*}{$2000-2010$} & PCA & 3 & 0.71 & 0.71 \\
\hline & GA-DFA 1 & 3 & 0.81 & 0.69 \\
\hline & GA-DFA 2 & 4 & 0.76 & 0.74 \\
\hline & GA-DFA 3 & 5 & 0.77 & 0.77 \\
\hline & Average & & 0.79 & 0.75 \\
\hline \multirow{7}{*}{$1958-2000$} & KW-H & 3 & 0.91 & 0.81 \\
\hline & PCA & 3 & 0.79 & 0.83 \\
\hline & GA-DFA 1 & 3 & 0.90 & 0.90 \\
\hline & GA-DFA 2 & 4 & 0.90 & 0.90 \\
\hline & GA-DFA 3 & 5 & 0.91 & 0.91 \\
\hline & Average & & 0.88 & 0.87 \\
\hline & KW-H & 3 & 0.87 & 0.84 \\
\hline \multirow{5}{*}{ Pre-1958 } & PCA & 3 & 0.88 & 0.88 \\
\hline & GA-DFA 1 & 3 & 0.88 & 0.88 \\
\hline & GA-DFA 2 & 4 & 0.91 & 0.91 \\
\hline & GA-DFA 3 & 5 & 0.90 & 0.89 \\
\hline & Average & & 0.89 & 0.88 \\
\hline
\end{tabular}

a-5 000 model iterations

b-30 000 model iterations 
Table 9. Summary of the predicted median source proportions (\%). Single values represent the overall relative frequency-weighted average medians; ranges represent the full uncertainty in the median contributions predicted by the Monte Carlo repeat runs of the mass balance model.

\begin{tabular}{|c|c|c|c|c|c|}
\hline \multirow{2}{*}{$\begin{array}{l}\text { Sediment } \\
\text { Site } 1\end{array}$} & \multirow[t]{2}{*}{ Signature } & \multicolumn{2}{|l|}{5000} & \multicolumn{2}{|l|}{30000} \\
\hline & & Surface & Subsurface & Surface & Subsurface \\
\hline \multirow[t]{11}{*}{$2000-2010$} & KW-H & 49 & 51 & 49 & 51 \\
\hline & & $0-100$ & $0-100$ & $0-100$ & $0-100$ \\
\hline & PCA & 45 & 55 & 45 & 55 \\
\hline & & $0-100$ & $0-100$ & $0-100$ & $0-100$ \\
\hline & GA-DFA 1 & 75 & 25 & 75 & 25 \\
\hline & & $0-100$ & $0-100$ & $0-100$ & $0-100$ \\
\hline & GA-DFA 2 & 67 & 33 & 67 & 33 \\
\hline & & $0-100$ & $0-100$ & $0-100$ & $0-100$ \\
\hline & GA-DFA 3 & 78 & 22 & 78 & 22 \\
\hline & & $0-100$ & $0-100$ & $0-100$ & $0-100$ \\
\hline & Weighted average ${ }^{a}$ & 49 & 51 & 49 & 51 \\
\hline \multirow[t]{11}{*}{$1958-2000$} & $\mathrm{KW}-\mathrm{H}$ & 52 & 48 & 52 & 48 \\
\hline & & $0-100$ & $0-100$ & $0-100$ & $0-100$ \\
\hline & PCA & 46 & 54 & 46 & 54 \\
\hline & & $0-100$ & $0-100$ & $0-100$ & $0-100$ \\
\hline & GA-DFA 1 & 55 & 45 & 55 & 45 \\
\hline & & $0-100$ & $0-100$ & $0-100$ & $0-100$ \\
\hline & GA-DFA 2 & 50 & 50 & 49 & 51 \\
\hline & & $0-100$ & $0-100$ & $0-100$ & $0-100$ \\
\hline & GA-DFA 3 & 47 & 53 & 48 & 52 \\
\hline & & $0-100$ & $0-100$ & $0-100$ & $0-100$ \\
\hline & Weighted average ${ }^{a}$ & 49 & 51 & 49 & 51 \\
\hline \multirow{12}{*}{$\begin{array}{l}\text { Site 2 } \\
2000-2010\end{array}$} & & & & & \\
\hline & KW-H & 25 & 75 & 26 & 74 \\
\hline & & $0-100$ & $0-100$ & $0-100$ & $0-100$ \\
\hline & PCA & 53 & 47 & 53 & 47 \\
\hline & & $0-100$ & $0-100$ & $0-100$ & $0-100$ \\
\hline & GA-DFA 1 & 18 & 82 & 31 & 69 \\
\hline & & $0-100$ & $0-100$ & $0-100$ & $0-100$ \\
\hline & GA-DFA 2 & 30 & 70 & 48 & 52 \\
\hline & & $0-100$ & $0-100$ & $0-100$ & $0-100$ \\
\hline & GA-DFA 3 & 29 & 71 & 30 & 70 \\
\hline & & $0-100$ & $0-100$ & $0-100$ & $0-100$ \\
\hline & Weighted average ${ }^{a}$ & 31 & 69 & 43 & 57 \\
\hline \multirow[t]{6}{*}{$1958-2000$} & $\mathrm{KW}-\mathrm{H}$ & 61 & 39 & 60 & 40 \\
\hline & & $0-100$ & $0-100$ & $0-100$ & $0-100$ \\
\hline & PCA & 66 & 34 & 64 & 36 \\
\hline & & $0-100$ & $0-100$ & $0-100$ & $0-100$ \\
\hline & GA-DFA 1 & 58 & 42 & 58 & 42 \\
\hline & & $0-100$ & $0-100$ & $0-100$ & $0-100$ \\
\hline
\end{tabular}




\begin{tabular}{llllll} 
& GA-DFA 2 & 74 & 26 & 74 & 26 \\
& & $30-100$ & $0-75$ & $0-100$ & $0-100$ \\
GA-DFA 3 & 75 & 25 & 75 & 25 \\
& & $30-100$ & $0-75$ & $0-100$ & $0-100$ \\
& Weighted average & \\
& & $\mathbf{6 1}$ & $\mathbf{3 9}$ & $\mathbf{6 3}$ & $\mathbf{3 7}$ \\
& 46 & 54 & 46 & 54 \\
& KW-H & $30-100$ & $0-75$ & $0-100$ & $0-100$ \\
& PCA & 45 & 55 & 46 & 54 \\
& & $30-100$ & $0-75$ & $0-100$ & $0-100$ \\
GA-DFA 1 & 45 & 55 & 48 & 52 \\
& & $30-100$ & $0-75$ & $0-100$ & $0-100$ \\
GA-DFA 2 & 63 & 37 & 63 & 37 \\
& & $30-100$ & $0-75$ & $0-100$ & $0-100$ \\
GA-DFA 3 & 58 & 42 & 58 & 42 \\
& & $30-100$ & $0-75$ & $0-100$ & $0-100$ \\
\hline
\end{tabular}

${ }^{a}$ Estimated using a weighting factor combining the GOF (Eq. 5) and \% discriminatory power for each signature. 
Site 1

2000-2010

KW-H

Surface

Subsurface
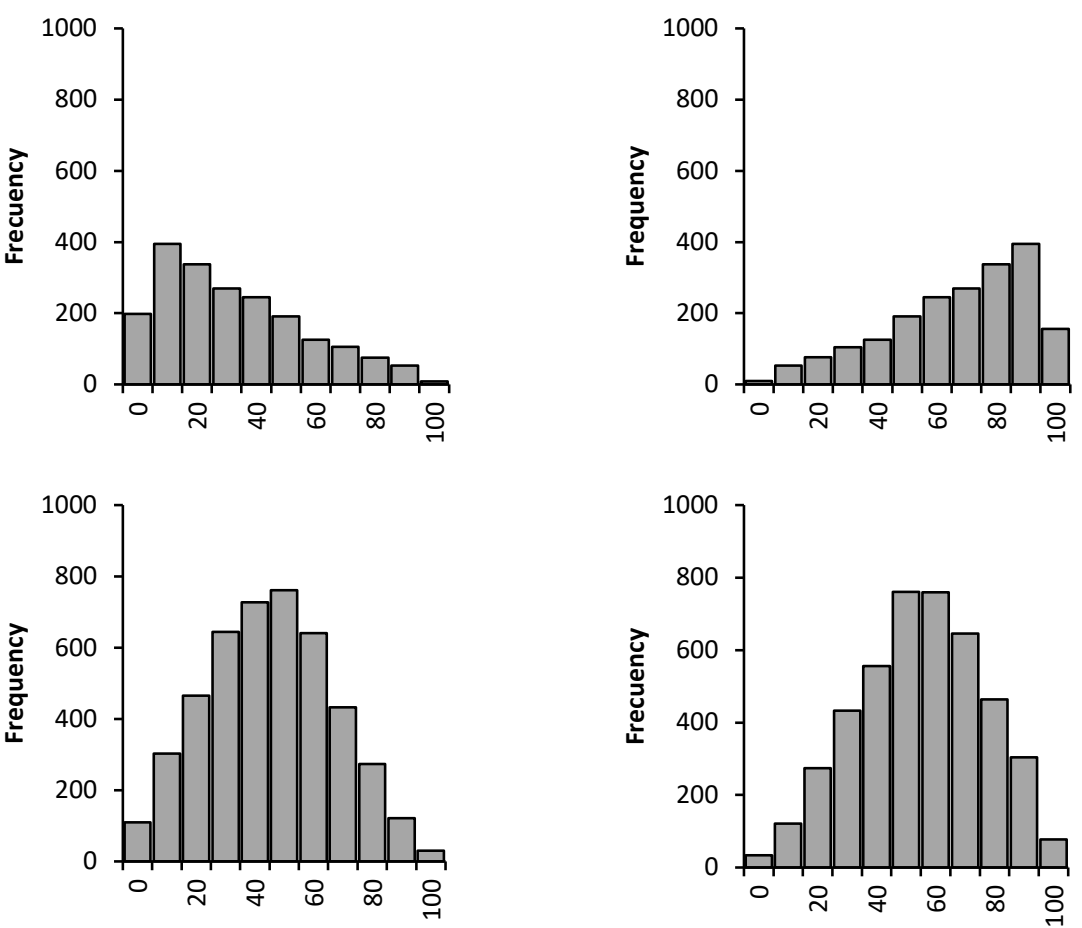

GA-DFA

Run 1
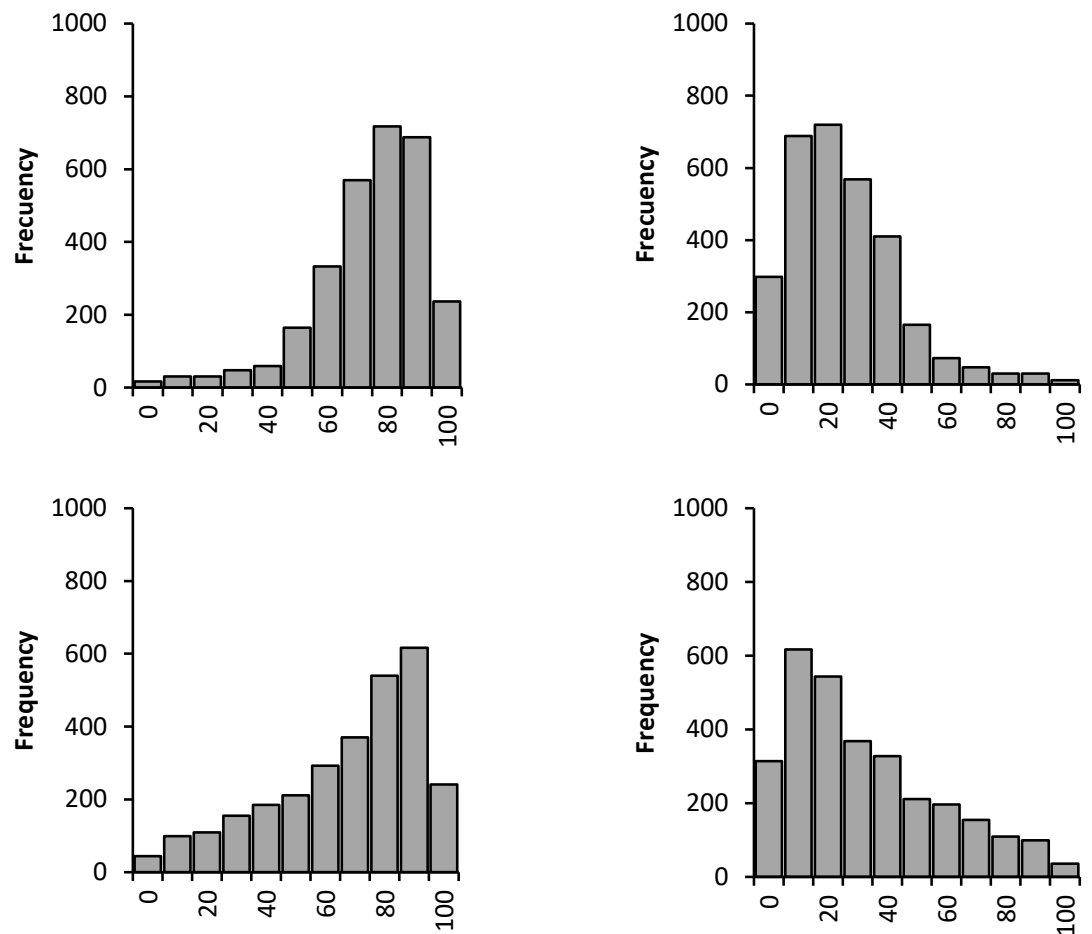
GA-DFA Run 3

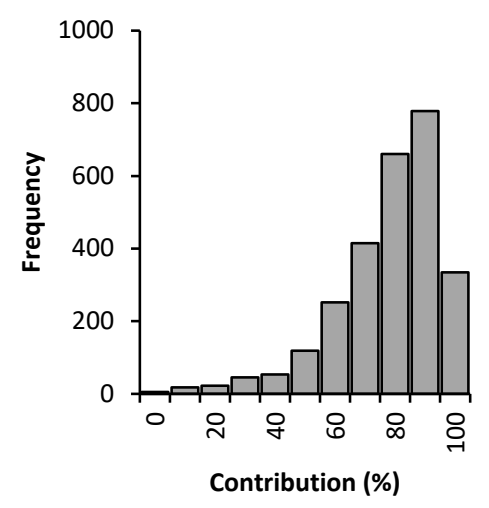

1958-2000

KW-H

PCA

GA-DFA

Run 1
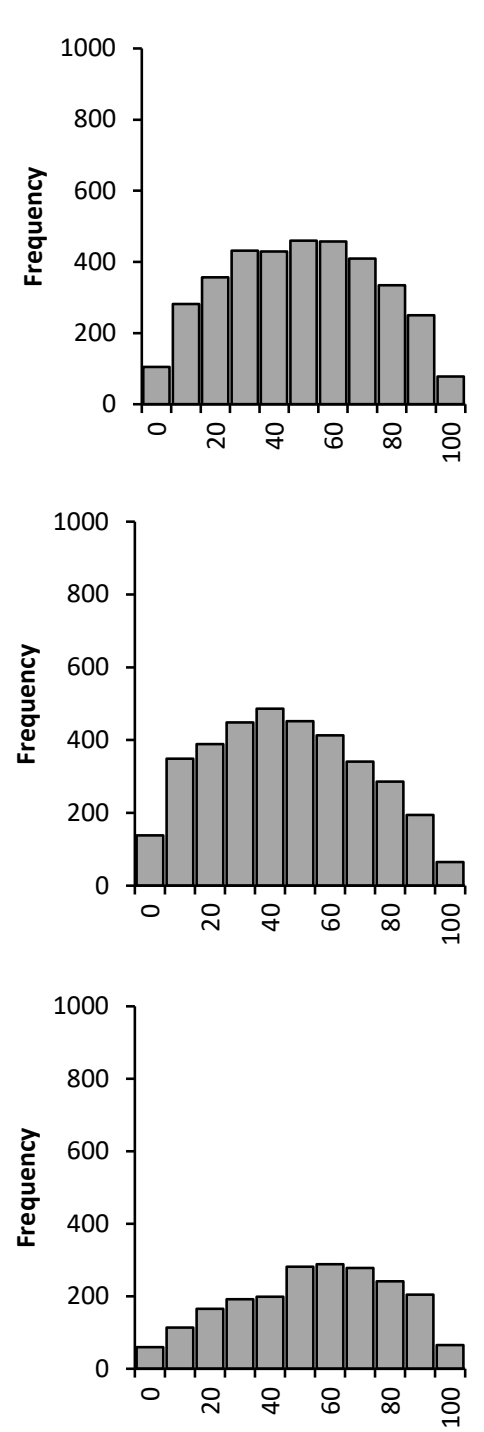
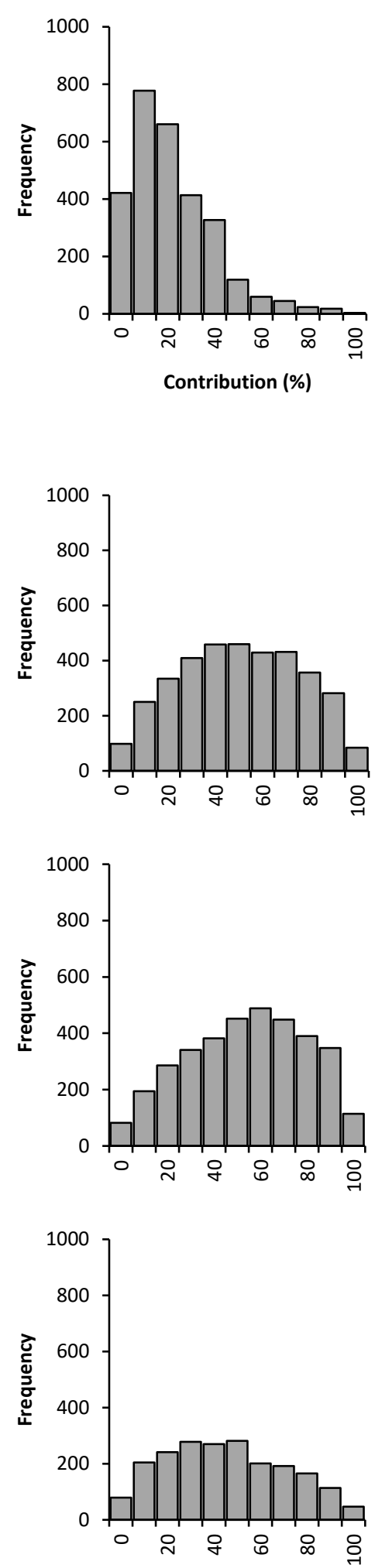
GA-DFA

Run 2

GA-DFA

Run 3

KW-H

Site 2

2000-2010
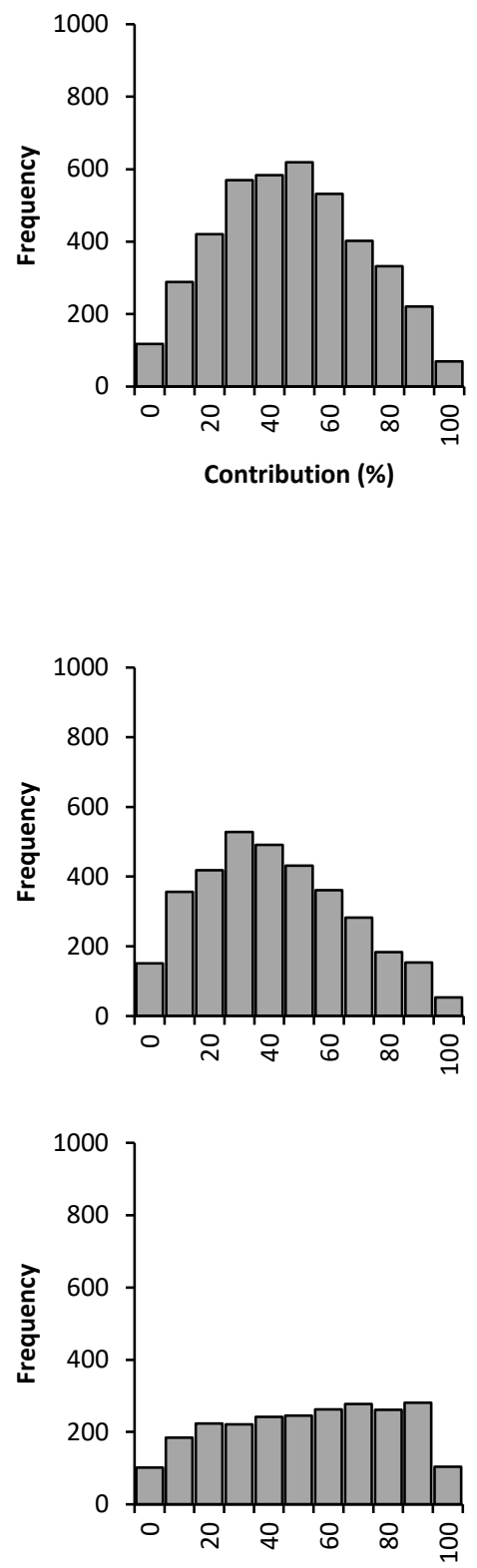
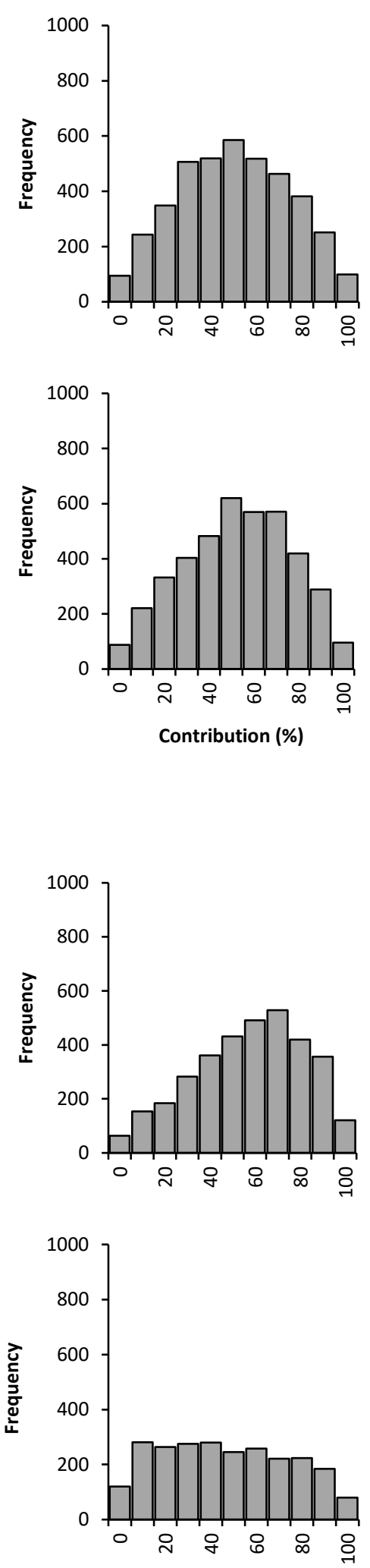
GA-DFA

Run 1

GA-DFA

Run 2

GA-DFA

Run 3

KW-H
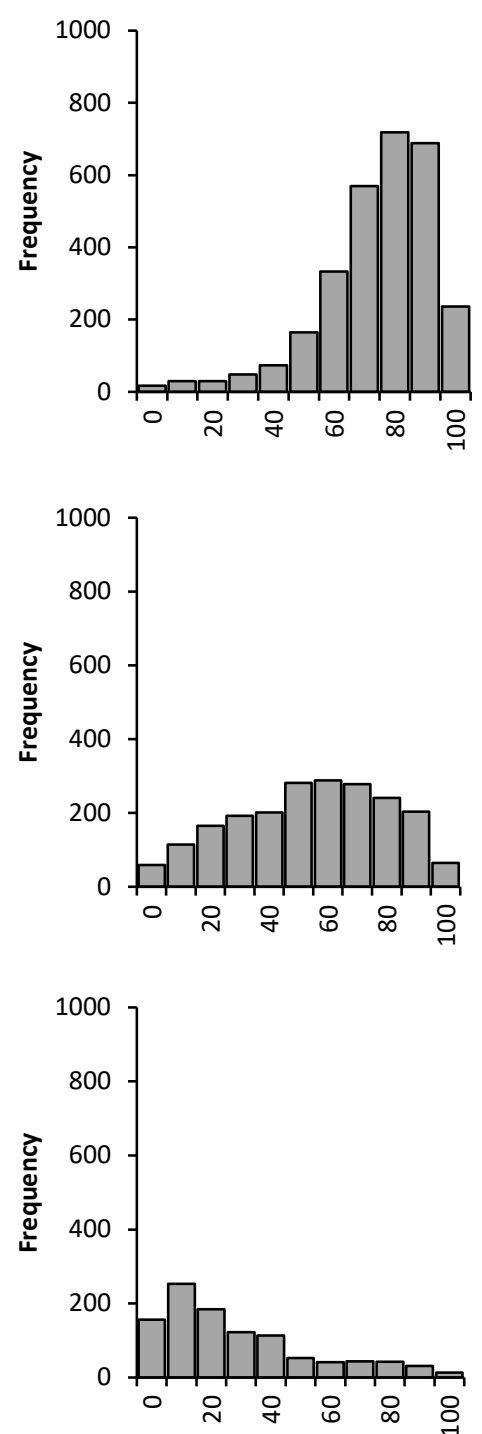

1958-2000

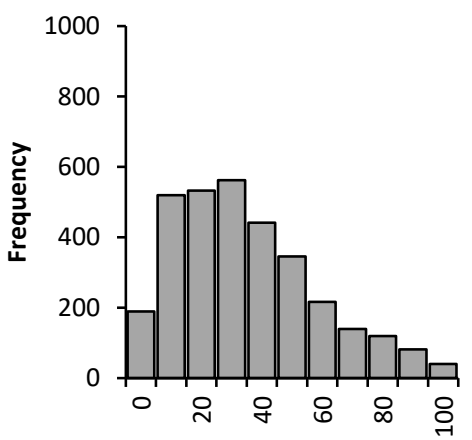

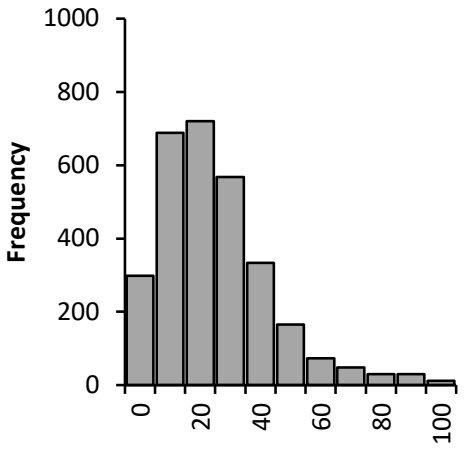
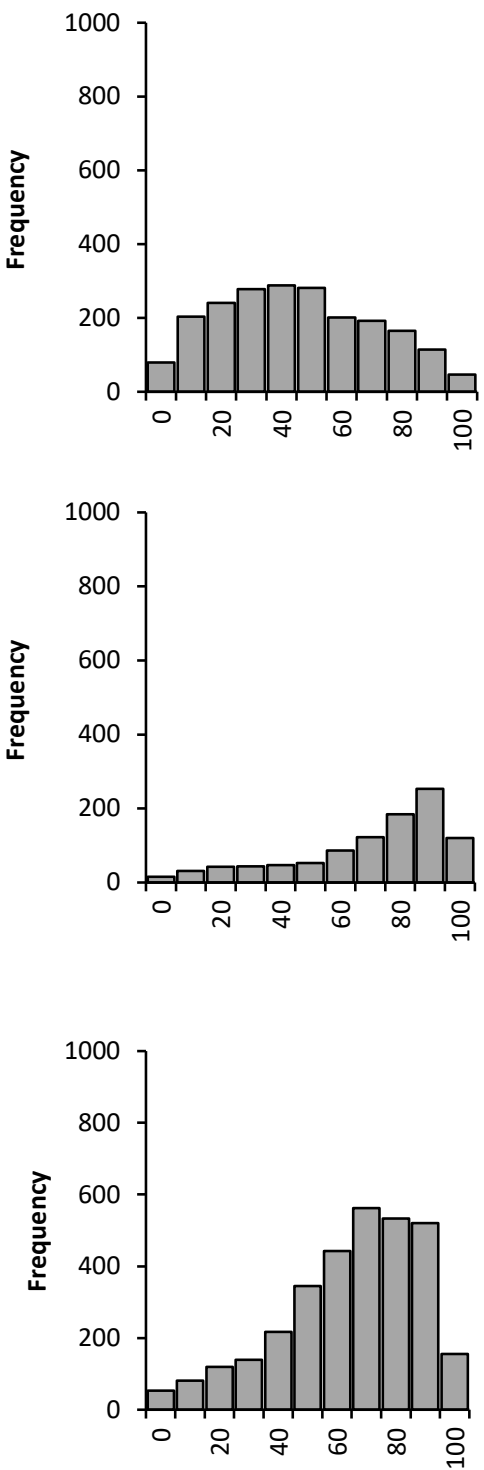
PCA
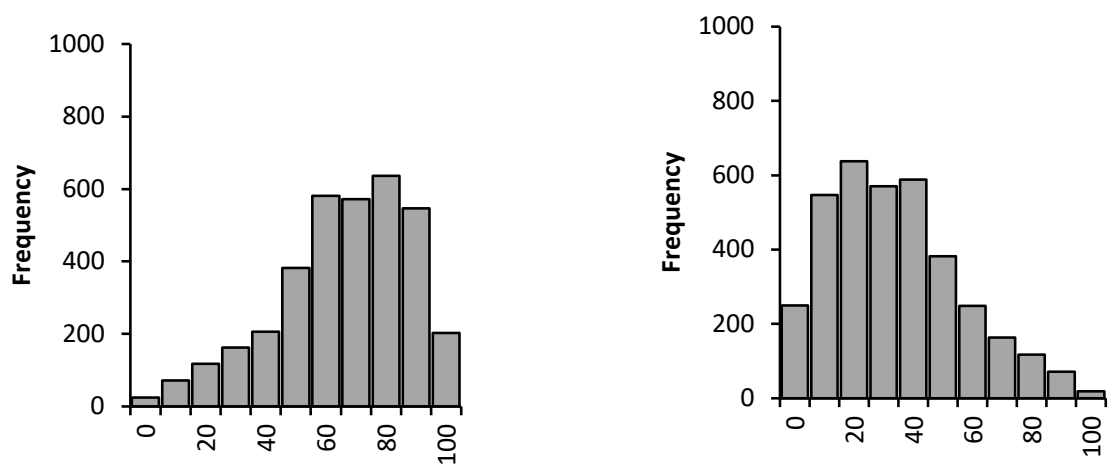

GA-DFA

Run 1
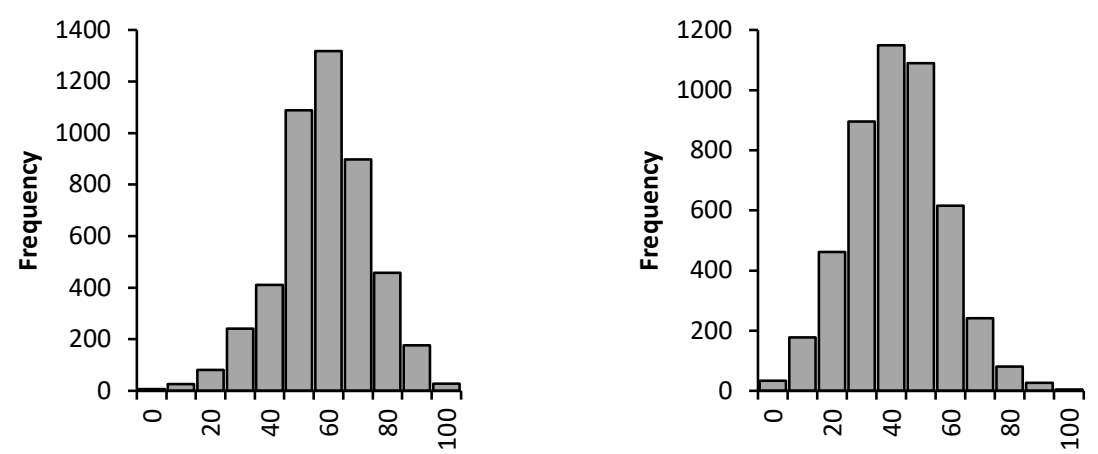

GA-DFA

Run 2
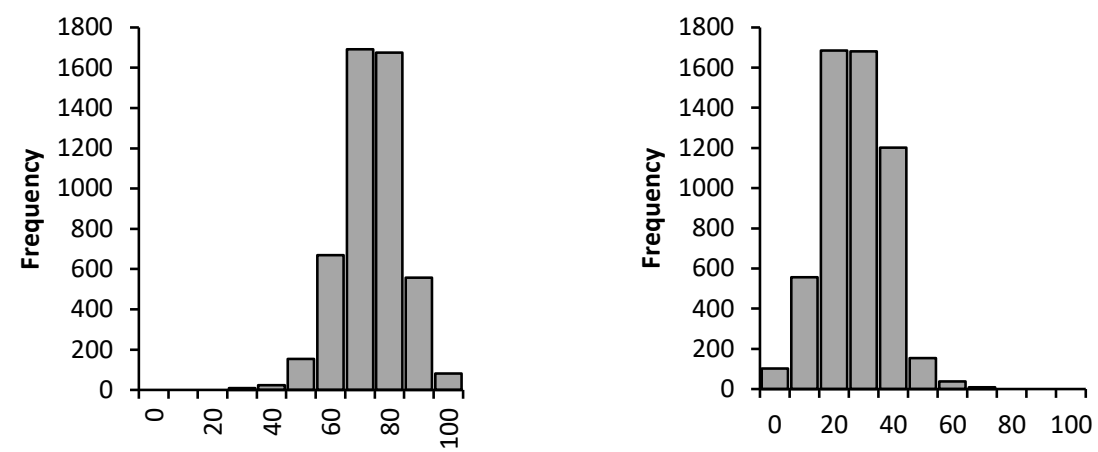

GA-DFA

Run 3
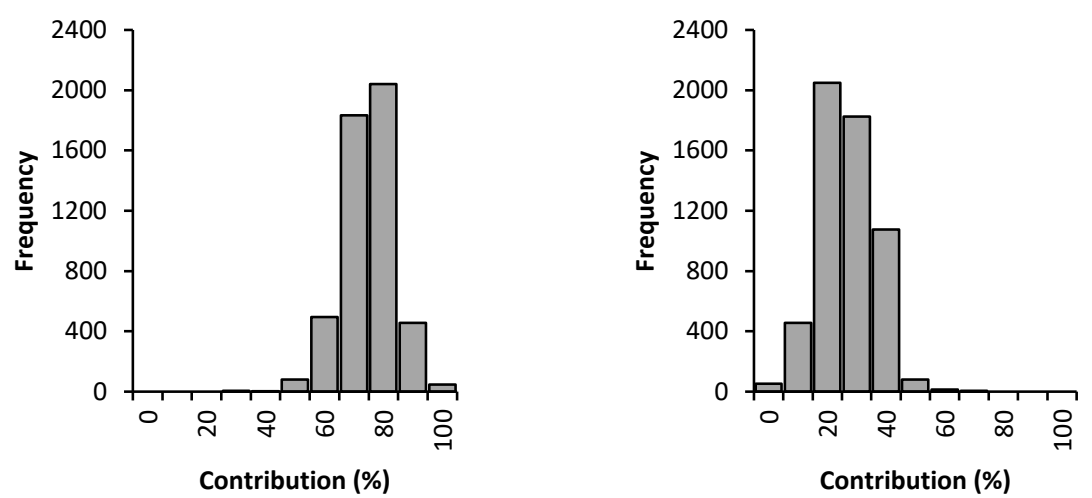

Pre-1958 
KW-H
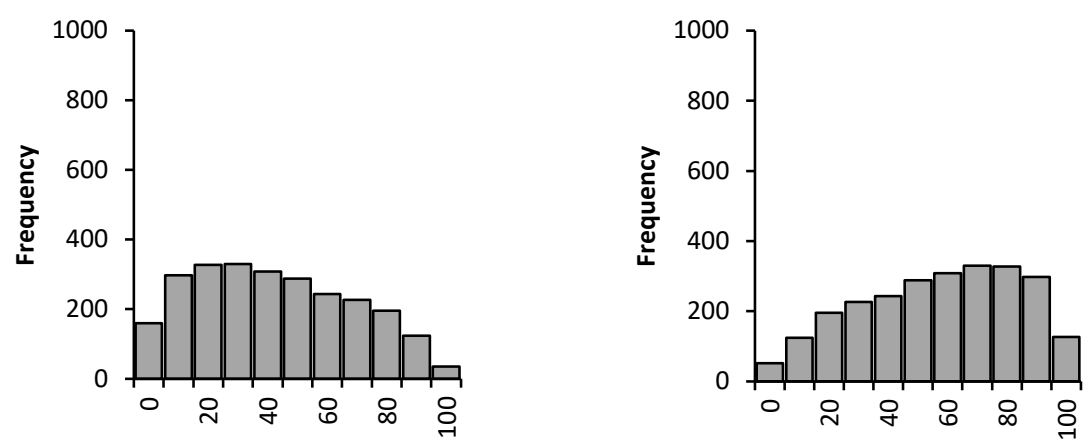

PCA
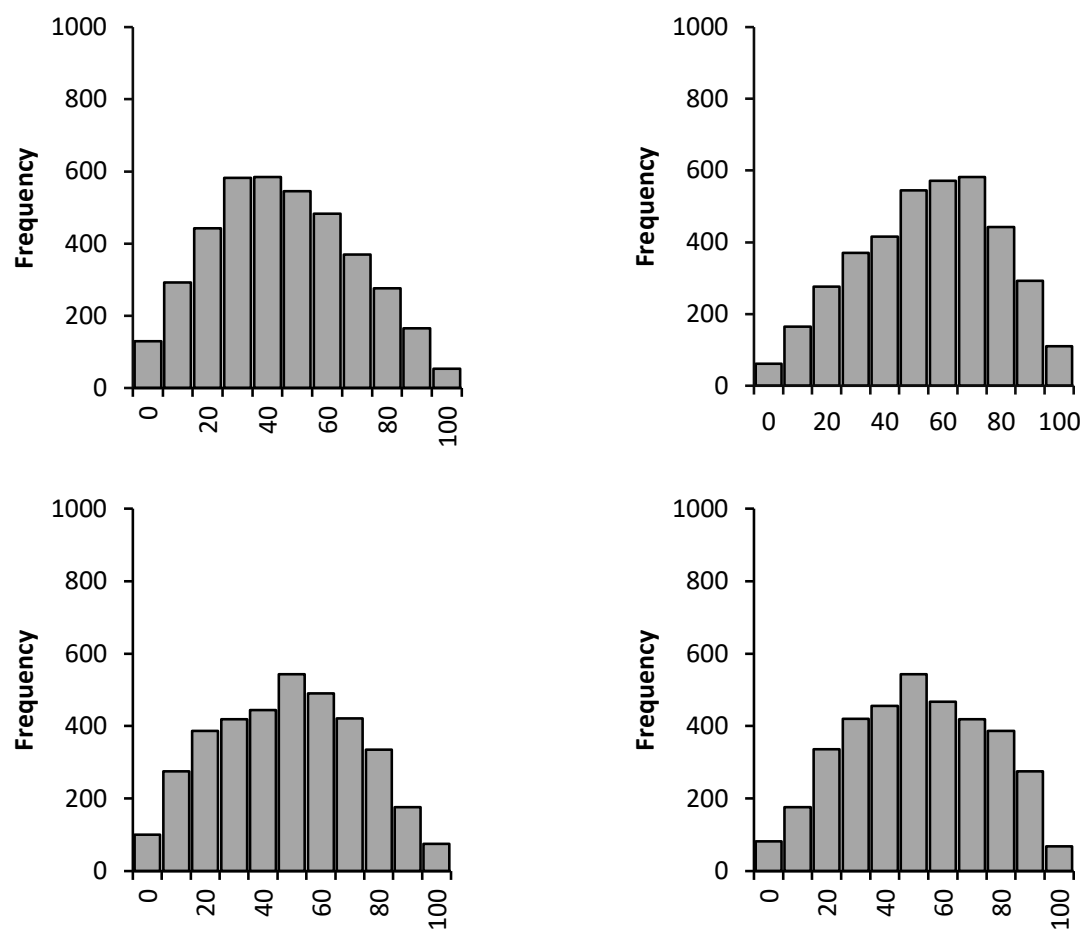

GA-DFA

Run 1
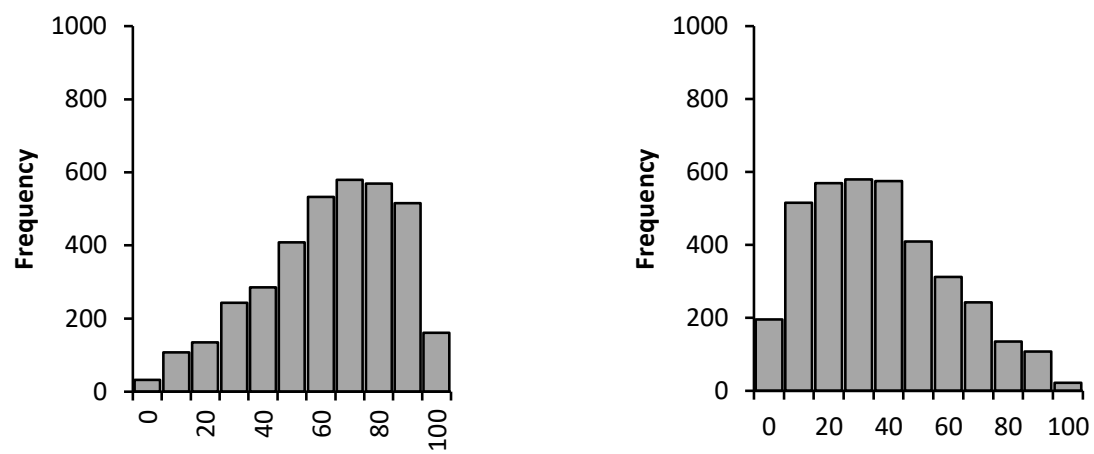

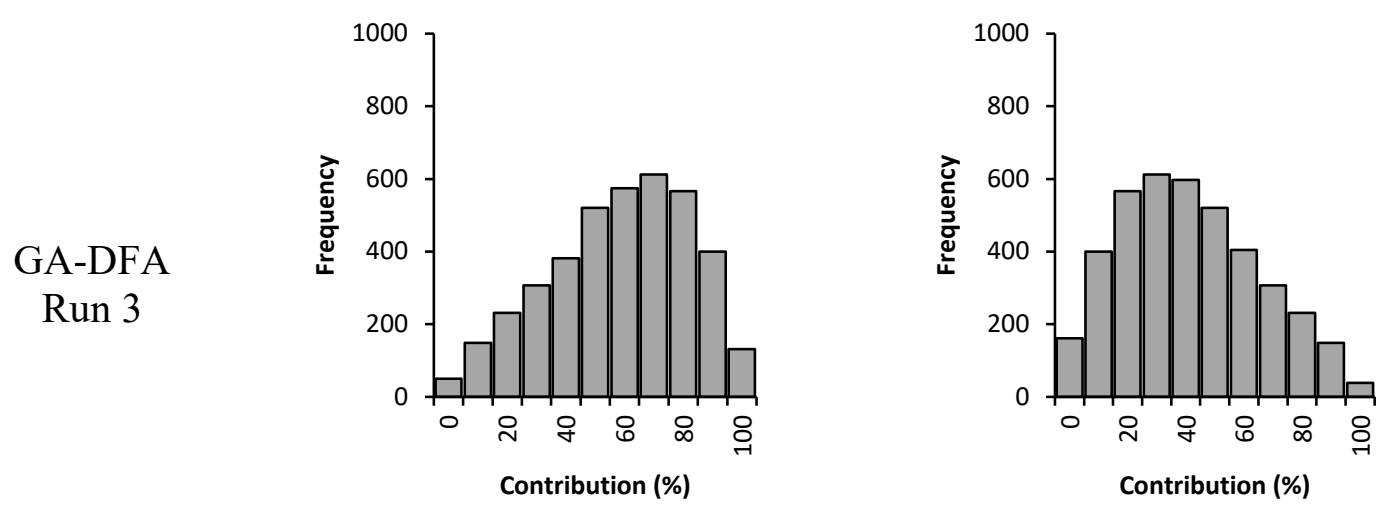

Figure 4. Probability density functions (pdfs) for the predicted median relative contributions from surface and subsurface sources in the study catchment.

For floodplain core Site 1 for the periods 2000-2010 and 1958-2000, there was perfect coincidence in the model solutions using the sets of 5000 and 30000 , both yielding surface and subsurface weighted average median contributions of $49 \%$ and $51 \%$, respectively. For the floodplain core Site 2, the weighted average median contributions from surface and subsurface sources were estimated at $31 \%$ and $69 \%$ respectively using 5000 model iterations and $43 \%$ and $57 \%$ respectively, using 30000 model iterations. For the 1958-2000 period, surface and subsurface sources respectively contributed $61 \%$ and $39 \%$ using 5000 model iterations and $63 \%$ and $37 \%$ using 30000 iterations. The model solutions using the sets of 5000 and 30000 iterations for the period before 1958 yielded the same weighted average median contributions of $52 \%$ and $48 \%$, respectively, for surface and subsurface sediment sources. These results suggest that for this dataset, the estimated source contributions are not highly sensitive to the number of un-mixing model iterations used in the Monte Carlo routine.

\subsection{Source apportionment and interpretation}

The results shown in Figure 5 (only results of 5000 iterations are shown) reflect the final weighted median contributions from surface and subsurface sources to the sediment cores collected at two sites $35 \mathrm{~m}$ apart on the same floodplain. While increased sediment contributions from subsurface sources is an indicator of gully or stream bank erosion and that from surface sources reflects sheet and inter-rill erosion (Krause et al., 2003; Hancock et al., 2013; Wallbrink et al., 1996), previous research in the catchment has shown that rill and gully erosion were increasingly becoming more severe (Kakembo and Rowntree, 2003; Kakembo et al., 2009). Thus subsurface sediment sources are most likely to be indicative of rill and gully erosion rather than stream bank erosion. For Site 1, the sediment 
record representing the period from 1958 to 2000 and 2000-2010 shows no change in sediment contribution from surface and subsurface processes. On the other hand, at Site 2, the sediment record reflects a decrease in sediment mobilized by rilling and gullying from $48 \%$ before 1958 to $39 \%$ of the deposited sediment between 1958 and 2000. However, between 2000 and 2010 there was a sudden rise in the contribution of sediment from rill and gully erosion sites (69\%). This abrupt change in sediment source is surprising and should be interpreted in the context of the high uncertainty indicated by the lower model GOF for the sediment samples represented by this period (Table 7).
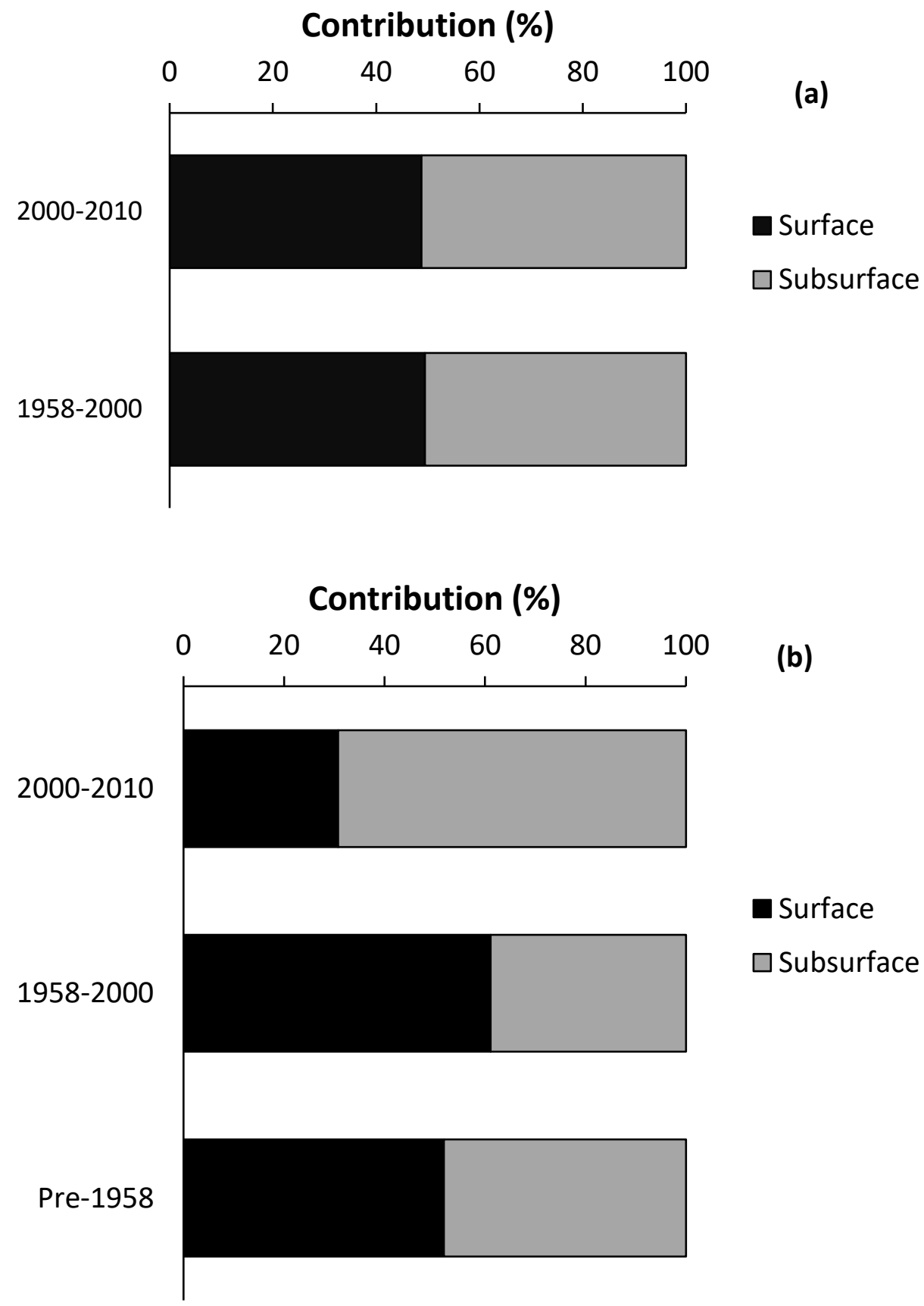
Figure 5. The overall estimate of the relative frequency-weighted average median contributions from surface and subsurface sources to floodplain sediment samples collected from Site 1 (a) and Site 2 (b).

The large differences in the predicted sediment source estimates for each time period between the two proximal sediment cores in the same floodplain reflects some of the uncertainties associated with the use of floodplain sediment cores in sourcing investigations. Sediment deposition on floodplains is known to exhibit high spatial variability in response to variations in micro-topography, vegetation and distance from the main river channel (Owens et al., 1999; Walling and Bradley, 1989; Walling and $\mathrm{He}, 1997)$. Areas closer to the river channel may experience slightly higher deposition rates while areas near the floodplain margin are likely to have lower deposition rates (Owens et al., 1999). The first site was located at a slightly higher landscape position $(\sim 30 \mathrm{~cm})$ than Core 2 , which was additionally situated near to the site of a former channel. Thus, the overbank deposition at the sites of the two cores could have happened at different times and been associated with different flood events. Overall, the results underpin the need to take replicate cores from depositional sinks to capture any spatial variation in the properties of deposited fine-grained sediment and indeed the chronology of that deposition.

The only long term published research from the study catchment and surrounding areas that documents the soil erosion status since 1938 (Kakembo and Rowntree, 2003) indicated that only after the mid-1970s did severe forms of soil erosion and gully erosion become widespread, coinciding with increased land abandonment and a period of extreme rainfall events. Thus, the role of subsurface processes is not expected to be dominant before the mid-1970s. A recent study (Manjoro et al., 2012a) covering the period 1998-2008 showed that severe soil erosion including gully erosion increased simultaneously with the increase in bush encroachment on representative hill slopes in the upper part of the study catchment. Recent field observations in the study catchment have indicated that although gully erosion is very severe, especially in the upper part of the catchment, in spatial terms the area affected is less than that affected by surface processes. Thus, the sudden change in sediment source predicted by the tracing results for recent sediment at Site 2 do not agree with alternative evidence for the study area. This example reminds users of the need to interpret source fingerprinting estimates on the context of alternative data sources for soil erosion and sediment delivery.

\section{Conclusion}

This study, based on a single catchment in South Africa, has explored the uncertainties in sediment source fingerprinting associated with increasing the number of properties in composite 
signatures, increasing the number of repeat model iterations and the use of two sediment profiles collected from the same floodplain sediment sink. Although some research has indicated that increasing the number of fingerprints in mass balance modelling will improve the GOF with measured sediment tracer values, we found that increasing the number of individual fingerprint properties included in the composite signature did not result in a higher model GOF. Given the potential counterintuitive performance of a widely-used GOF estimator, we adopted an alternative. It was also noted that increasing the number of repeat model iterations did not significantly change uncertainty ranges in modelled source proportions nor improve the GOF. Accordingly, the model results based on 5000 iterations were used to assess the importance of subsurface processes in the study catchment. The temporal patterns of sediment source contributions from surface and subsurface processes predicted for the two sediment cores were very different despite the cores being collected in close proximity from the same floodplain sink. Although only two sediment cores were used in this study, the results show that sediment apportionment results can vary even using historical sediment collected in close proximity. Although floodplain sediment has been proven to be an important source of proxy historical data for environmental processes including soil erosion (Collins et al., 1997b; Owens et al., 1999), the spatial variability of sediment deposition on floodplains means that floodplain cores collected from the same depositional unit may produce different sediment records. The sediment source contribution estimates from this study are not consistent with the available published data on erosion processes and thereby highlight the potential limitations associated with using floodplain cores to reconstruct catchment erosion processes and associated sediment source contributions in complete independence of alternative data sources.

\section{Acknowledgements}

The mineral magnetic analysis was undertaken in a lab financed by the National Research Foundation (NRF) at Geography Department of Rhodes University while the gamma spectrometry was done at Rhodes University and the University of Coventry. The study was funded by NRF grant awarded to $\mathrm{KM}$ and VK. The manuscript was prepared while MM was on a research visit in the Department of Sustainable Soil and Grassland Systems at Rothamsted Research, UK, financed by British Council Newton Fund: Researcher Links Travel Grant. 


\section{References}

Agricultural Research Council (ARC). 2010. Climate data for Peddie Mag climate station-10876. Grahamstown.

Amos, K.J., Croke, J.C., Timmers, H., Owens, P.N., Thompson, C. (2009). The application of caesium-137 measurements to investigate floodplain deposition in a large semi-arid catchment in Queensland, Australia. Earth Surface Processes and Landforms 34 (4), 515-529.

Belmont, P., Willenbring, J. K., Schottler, S. P., Marquard, J., Kumarasamy, K., and Hemmis, J. M. (2014). Toward generalizable sediment fingerprinting with tracers that are conservative and nonconservative over sediment routing timescales. Journal of soils and sediments, 14(8), 1479-1492.

Boardman, J., Foster, I.D.L. (2008). Badland and gully erosion in the Karoo, South Africa. Journal of Soil and Water Conservation 63, 4.

Boardman, J., Foster, I., Rowntree, K., Mighall, T., \& Gates, J. (2010). Environmental stress and landscape recovery in a semi-arid area, the Karoo, South Africa. Scottish Geographical Journal, 126(2), 64-75.

Boardman, J., Parsons, A. J., Holland, R., Holmes, P. J., and Washington, R. (2003). Development of badlands and gullies in the Sneeuberg, Great Karoo, South Africa. Catena, 50(2), 165-184.

Bottrill, L. J., Walling, D. E., and Leeks, G. J. L. (2000). Using recent overbank deposits to investigate contemporary sediment sources in larger river basins. Tracers in geomorphology. Chichester: Wiley, $369-87$.

Collins, A. L., and Walling, D. E. (2007). Sources of fine sediment recovered from the channel bed of lowland groundwater-fed catchments in the UK. Geomorphology, 88(1), 120-138.

Collins, A. L., Walling, D. E., and Leeks, G. J. L. (1996). Composite fingerprinting of the spatial source of fluvial suspended sediment: a case study of the Exe and Severn River basins, United Kingdom. Géomorphologie: relief, processus, environnement, 2(2), 41-53.

Collins, A. L., Walling, D. E., and Leeks, G. J. L. (1997a). Source type ascription for fluvial suspended sediment based on a quantitative composite fingerprinting technique. Catena, 29(1), 1-27.

Collins, A. L., Walling, D. E., and Leeks, G. J. L. (1997b). Use of the geochemical record preserved in floodplain deposits to reconstruct recent changes in river basin sediment sources. Geomorphology, 19(1), 151-167.

Collins, A. L., Walling, D. E., and Leeks, G. J. L. (1998). Use of composite fingerprints to determine the provenance of the contemporary suspended sediment load transported by rivers. Earth surface processes and landforms, 23(1), 31-52. 
Collins, A. L., Walling, D. E., Stroud, R. W., Robson, M., and Peet, L. M. (2010a). Assessing damaged road verges as a suspended sediment source in the Hampshire Avon catchment, southern United Kingdom. Hydrological Processes, 24(9), 1106-1122.

Collins, A. L., Walling, D. E., Webb, L., and King, P. (2010b). Apportioning catchment scale sediment sources using a modified composite fingerprinting technique incorporating property weightings and prior information. Geoderma, 155(3), 249-261.

Collins, A. L., Zhang, Y. S., Hickinbotham, R., Bailey, G., Darlington, S., Grenfell, S. E., Evans, R., Blackwell, M. (2013). Contemporary fine-grained bed sediment sources across the River Wensum Demonstration Test Catchment, UK. Hydrological Processes, 27(6), 857-884.

Collins, A. L., Zhang, Y., McChesney, D., Walling, D. E., Haley, S. M., and Smith, P. (2012a). Sediment source tracing in a lowland agricultural catchment in southern England using a modified procedure combining statistical analysis and numerical modelling. Science of the Total Environment, 414, 301317.

Collins, A. L., Zhang, Y., Walling, D. E., Grenfell, S. E., Smith, P., Grischeff, J., Locke,A., Sweetapple, A., Brogden,D. (2012b). Quantifying fine-grained sediment sources in the River Axe catchment, southwest England: application of a Monte Carlo numerical modelling framework incorporating local and genetic algorithm optimisation. Hydrological Processes, 26(13), 1962-1983.

Dearing, J. (1999). Environmental magnetic susceptibility using the Bartington MS2 System, Chi Publishing, Kenilworth, UK.

D’Haen, K., Verstraeten, G., and Degryse, P. (2012). Fingerprinting historical fluvial sediment fluxes. Progress in Physical Geography, 36(2), 154-186.

De Vente, J., and Poesen, J. (2005). Predicting soil erosion and sediment yield at the basin scale: scale issues and semi-quantitative models. Earth-Science Reviews, 71(1), 95-125.

Devereux, O. H., Prestegaard, K. L., Needelman, B. A., and Gellis, A. C. (2010). Suspended-sediment sources in an urban watershed, Northeast Branch Anacostia River, Maryland. Hydrological processes, 24(11), 1391-1403.

Dollar, E.S.J., and Rowntree, K.M. (1995). Hydroclimatic trends, sediment sources and geomorphic response in the Bell River catchment, Eastern Cape, Drakensberg, South Africa. South African Geographical Journal, 77, 21-32.

Evrard, O., Navratil, O., Ayrault, S., Ahmadi, M., Némery, J., Legout, C., Lefèvre, I., Poirel, A., Bonté, P., and Esteves, M. (2011). Combining suspended sediment monitoring and fingerprinting to determine the spatial origin of fine sediment in a mountainous river catchment. Earth Surface Processes and Landforms, 36(8), 1072-1089. 
Foster, I.D.L., Boardman, J., Keay-Bright, J. (2007). Sediment tracing and environmental history for two small catchments, Karoo Uplands, South Africa. Geomorphology, 90: 126-143.

Foster, I.D.L., Boardman, J., Keay-Bright, J., and Meadows, M.E.(2005). Land degradation and sediment dynamics in the South African Karoo. International Association of Hydrological Sciences Publication 292, 207-213.

Foster IDL, Lees JA. 2000. Tracers in geomorphology: theory and applications in tracing fine particulate sediments. In Tracers in Geomorphology, Foster IDL (Ed.). Wiley: Chichester; 3-20.

Foster, I. D., Lees, J. A., Owens, P. N., and Walling, D. E. (1998). Mineral magnetic characterization of sediment sources from an analysis of lake and floodplain sediments in the catchments of the Old Mill Reservoir and Slapton Ley, South Devon, UK. Earth Surface Processes and Landforms, 23(8), 685703.

Foster, I. D., Rowntree, K. M., Boardman, J., and Mighall, T. M. (2012). Changing sediment yield and sediment dynamics in the Karoo uplands, South Africa; post-European impacts. Land Degradation and Development, 23(6), 508-522.

Fox, J. F., and Papanicolaou, A. N. (2008). Application of the spatial distribution of nitrogen stable isotopes for sediment tracing at the watershed scale. Journal of Hydrology, 358(1), 46-55.

Fryirs, K. A., Brierley, G. J., Preston, N. J., and Spencer, J. (2007). Catchment-scale (dis) connectivity in sediment flux in the upper Hunter catchment, New South Wales, Australia. Geomorphology 84(3), 297-316.

Fuller, I. C., and Marden, M. (2010). Rapid channel response to variability in sediment supply: cutting and filling of the Tarndale Fan, Waipaoa catchment, New Zealand. Marine Geology, 270(1), 45-54.

Gibbons, J. D., and Chakraborti, S. (2011). Nonparametric statistical inference (pp. 977-979). Springer Berlin Heidelberg.

Govers, G., and Poesen, J. (1988). Assessment of the interrill and rill contributions to total soil loss from an upland field plot. Geomorphology, 1(4), 343-354.

Gruszowski, K. E., Foster, I. D., Lees, J. A., and Charlesworth, S. M. (2003). Sediment sources and transport pathways in a rural catchment, Herefordshire, UK. Hydrological Processes, 17(13), 2665-2681.

Haddadchi, A., Ryder, D. S., Evrard, O., and Olley, J. (2013). Sediment fingerprinting in fluvial systems: review of tracers, sediment sources and mixing models. International Journal of Sediment Research, 28(4), 560-578.

Hancock, G. J., and Revill, A. T. (2013). Erosion source discrimination in a rural Australian catchment using compound-specific isotope analysis (CSIA).Hydrological Processes, 27(6), 923-932.

Hanvey, P. M., Dardis, G. F., and Beckedahl, H. R. (1991). Soil erosion on a sub-tropical coastal dune complex, Transkei, southern Africa. Geojournal, 23(1), 41-48. Harvey, A.M. (2001). Coupling 
between hillslopes and channels in upland fluvial systems: implications for landscape sensitivity, illustrated from the Howgill Fells, northwest England. Catena, 42, 225-250.

He, Q., and Walling, D. E. (1996). Interpreting particle size effects in the adsorption of 137 Cs and unsupported $210 \mathrm{~Pb}$ by mineral soils and sediments.Journal of Environmental Radioactivity, 30(2), 117-137.

Heiri, O., Lotter, A.F. and Lemcke, G., 2001. Loss on ignition as a method for estimating organic and carbonate content in sediments: reproducibility and comparability of results. Journal of Paleolimnology, 25 (1), 101-110.

Henderson, A. R. (2006). Testing experimental data for univariate normality. Clinica chimica acta, 366(1), 112-129.

Hollander, M., Wolfe, D. A., and Chicken, E. (2013). Nonparametric statistical methods. John Wiley and Sons.

Horowitz, A.J. (1991). A Primer on Sediment Trace Element Chemistry.Michigan. Lewis Publishers.

Hughes, A. O., Olley, J. M., Croke, J. C., and McKergow, L. A. (2009). Sediment source changes over the last 250 years in a dry-tropical catchment, central Queensland, Australia. Geomorphology, 104(3), $262-275$.

Jarvis, R. M., and Goodacre, R. (2005). Genetic algorithm optimization for pre-processing and variable selection of spectroscopic data. Bioinformatics, 21(7), 860-868.

Jolliffe, I. (2002). Principal component analysis. John Wiley and Sons, Ltd.

Kakembo, V. (2009). Vegetation patchiness and implications for landscape function: The case of Pteronia incana invader species in Ngqushwa Rural Municipality, Eastern Cape, South Africa. Catena, 77(3), 180-186.

Kakembo, V., and Rowntree, K. M. (2003). The relationship between land use and soil erosion in the communal lands near Peddie Town, Eastern Cape, South Africa. Land Degradation and Development, 14(1), 39-49.

Kakembo, V., Xanga, W. W., and Rowntree, K. (2009). Topographic thresholds in gully development on the hillslopes of communal areas in Ngqushwa Local Municipality, Eastern Cape, South Africa. Geomorphology, 110(3), 188-194.

Keay-Bright, J., and Boardman, J. (2009). Evidence from field-based studies of rates of soil erosion on degraded land in the central Karoo, South Africa.Geomorphology, 103(3), 455-465.

Keay-Bright, J., and Boardman, J. (2006). Changes in the distribution of degraded land over time in the central Karoo, South Africa. Catena, 67(1), 1-14.

Koiter, A. J., Lobb, D. A., Owens, P. N., Petticrew, E. L., Tiessen, K. H., and Li, S. (2013). Investigating the role of connectivity and scale in assessing the sources of sediment in an agricultural watershed in the 
Canadian prairies using sediment source fingerprinting. Journal of Soils and Sediments, 13(10), 16761691.

Koiter, A. J., Owens, P. N., Petticrew, E. L., and Lobb, D. A. (2013). The behavioural characteristics of sediment properties and their implications for sediment fingerprinting as an approach for identifying sediment sources in river basins. Earth-Science Reviews, 125, 24-42.

Krause, A.K., Franks, S.W., Kalma, J.D., Loughran, R.J and Rowan, J.S. (2003). Multi-parameter fingerprinting of sediment deposition in a small gullied catchment in SE Australia. Catena, 53, 327348.

Laceby, J. P., and Olley, J. (2015). An examination of geochemical modelling approaches to tracing sediment sources incorporating distribution mixing and elemental correlations. Hydrological Processes, 29(6), $1669-1685$.

Le Roux, J. J., and Sumner, P. D. (2012). Factors controlling gully development: comparing continuous and discontinuous gullies. Land Degradation and Development, 23(5), 440-449.

Leslie, C., and Hancock, G. J. (2008). Estimating the date corresponding to the horizon of the first detection of $137 \mathrm{Cs}$ and 239+ $240 \mathrm{Pu}$ in sediment cores. Journal of Environmental Radioactivity, 99(3), 483490.

Lilliefors, H. W. (1969). On the Kolmogorov-Smirnov test for the exponential distribution with mean unknown. Journal of the American Statistical Association, 64(325), 387-389.

Macklin, M. G., Ridgway, J., Passmore, D. G., and Rumsby, B. T. (1994). The use of overbank sediment for geochemical mapping and contamination assessment: results from selected English and Welsh floodplains. Applied Geochemistry, 9(6), 689-700.

Manjoro, M., Kakembo, V., and Rowntree, K. M. (2012a). Trends in soil erosion and woody shrub encroachment in Ngqushwa District, Eastern Cape Province, South Africa. Environmental management, 49(3), 570-579.

Manjoro, M., Rowntree, K., Kakembo, V., and Foster, I. D. (2012b). Gully fan morphodynamics in a small catchment in the Eastern Cape, South Africa. Land Degradation and Development, 23(6), 569-576.

Martinez-Carreras, N., Gallart, F., Iffly, J. F., Pfister, L., Walling, D. E., and Krein, A. (2008). Uncertainty assessment in suspended sediment fingerprinting based on tracer mixing models: a case study from Luxembourg. IAHS publication, 325, 94.

McKay, M. D., Beckman, R. J., and Conover, W. J. (1979). Comparison of three methods for selecting values of input variables in the analysis of output from a computer code. Technometrics, 21(2), 239-245.

Mckinley, R., Radcliffe, D., and Mukundan, R. (2013). A streamlined approach for sediment source fingerprinting in a Southern Piedmont watershed, USA.Journal of Soils and Sediments, 13(10), 17541769. 
Mills, A. J., and Cowling, R. M. (2006). Rate of carbon sequestration at two thicket restoration sites in the Eastern Cape, South Africa. Restoration Ecology, 14(1), 38-49.

Minella, J. P., Walling, D. E., and Merten, G. H. (2008). Combining sediment source tracing techniques with traditional monitoring to assess the impact of improved land management on catchment sediment yields. Journal of Hydrology, 348(3), 546-563.

Mukundan, R., Walling, D. E., Gellis, A. C., Slattery, M. C., and Radcliffe, D. E. (2012). Sediment Source Fingerprinting: Transforming From a Research Tool to a Management Tool1. JAWRA Journal of the American Water Resources Association, 48(6), 1241-1257.

Olley, J., and Caitcheon, G. (2000). Major element chemistry of sediments from the Darling-Barwon River and its tributaries: implications for sediment and phosphorus sources. Hydrological Processes, 14(7), 1159-1175.

Owens, P. N., Walling, D. E., and Leeks, G. J. (1999). Use of floodplain sediment cores to investigate recent historical changes in overbank sedimentation rates and sediment sources in the catchment of the River Ouse, Yorkshire, UK. Catena, 36(1), 21-47.

Owens, P. N., Walling, D. E., and Leeks, G. J. L. (2000). Tracing fluvial suspended sediment sources in the catchment of the River Tweed, Scotland, using composite fingerprints and a numerical mixing model. Tracers in geomorphology, 291-308.

Poesen, J., Vandekerckhove, L., Nachtergaele, J., Oostwoud Wijdenes, D., Verstraeten, G., van Wesemael, B., 2002. Gully erosion in dryland environments. In: Bull, L.J., Kirkby, M.J. (Eds.), Dryland Rivers. Wiley, Chichester, UK, pp. 229- 262.

Poleto, C., Merten, G. H., and Minella, J. P. (2009). The identification of sediment sources in a small urban watershed in southern Brazil: an application of sediment fingerprinting. Environmental technology, 30(11), 1145-1153.

Pulley, S., Foster, I. D., and Antunes, A. P. M. (2014). Fingerprinting fluvial sediments deposited over the last 100 years: a practical examination of the accuracy and uncertainty in the Nene basin, United Kingdom.

Pulley, S., Rowntree, K., and Foster, I. (2015). Conservatism of mineral magnetic signatures in farm dam sediments in the South African Karoo: the potential effects of particle size and post-depositional diagenesis. Journal of Soils and Sediments, 1-11.

Roddy, B. P. (2010). The use of the sediment fingerprinting technique to quantify the different sediment sources entering the Whangapoua Estuary, North Island, in New Zealand (Doctoral dissertation, University of Waikato). 
Rosewell, C. (1996). Rates of erosion and sediment transport in Australia. In Erosion and Sediment Yield: Global and Regional Perspectives: Proceedings of an International Symposium Held at Exeter, UK, from 15 to 19 July 1996 (No. 236, p. 139). IAHS.

Rousseeuw, P. J., and Croux, C. (1993). Alternatives to the median absolute deviation. Journal of the American Statistical association, 88(424), 1273-1283.

Rowan, J. S., Black, S., and Franks, S. W. (2012). Sediment fingerprinting as an environmental forensics tool explaining cyanobacteria blooms in lakes. Applied Geography, 32(2), 832-843.

Rowntree, K., and Foster, I. (2012). A reconstruction of historical changes in sediment sources, sediment transfer and sediment yield in a small, semi-arid Karoo catchment, semi-arid South Africa. Zeitschrift für Geomorphologie, Supplementary Issues, 56(1), 87-100.

Russell, M. A., Walling, D. E., and Hodgkinson, R. A. (2001). Suspended sediment sources in two small lowland agricultural catchments in the UK. Journal of Hydrology, 252(1), 1-24.

Sherriff, S. C., Franks, S. W., Rowan, J. S., Fenton, O., and Ó’hUallacháin, D. (2015). Uncertainty-based assessment of tracer selection, tracer non-conservativeness and multiple solutions in sediment fingerprinting using synthetic and field data. Journal of Soils and Sediments, 1-16.

Smith, H. G., and Blake, W. H. (2014). Sediment fingerprinting in agricultural catchments: a critical reexamination of source discrimination and data corrections. Geomorphology, 204, 177-191.

Smith, H. G., and Dragovich, D. (2008). Improving precision in sediment source and erosion process distinction in an upland catchment, south-eastern Australia. Catena, 72(1), 191-203.

Stone, M., Collins, A. L., Silins, U., Emelko, M. B., and Zhang, Y. S. (2014). The use of composite fingerprints to quantify sediment sources in a wildfire impacted landscape, Alberta, Canada. Science of the Total Environment, 473, 642-650.

Tabachnick, B.G., L.S. Fidell. (1996). Using Multivariate Statistics. Harper Collins College Publishers: New York.

Theuring, P., Rode, M., Behrens, S., Kirchner, G., and Jha, A. (2013). Identification of fluvial sediment sources in the Kharaa River catchment, Northern Mongolia. Hydrological Processes, 27(6), 845-856.

US EPA, 2007. Method 3051a: microwave-assisted acid digestion of sediments, sludges, soils and oils. Official Methods/US EPA methods, p. 846. Washington DC,

Vetter, S. (2007). Soil erosion in the Herschel district of South Africa: changes over time, physical correlates and land users' perceptions. African Journal of Range and Forage Science, 24(2), 77-86.

Walden, J. (1999). Remanance measurements, in: Walden, J., Oldfield, F., Smith, J. (Eds.); 1999. Environmental magnetism, a practical guide. Technical Guide No. 6. Quaternary Research Association. London, 1999, 243 pp. 
Wallbrink, P. J., and Olley, J. O. N. (2004). Sources of fine grained sediment in incised and un-incised channels, Jugiong Creek, NSW, Australia. In Sediment Transfer Through the Fluvial System: Proceedings of the International Symposium Held at Moscow, Russia, from 2 to 6 August, 2004 (No. 288, p. 165). International Assn of Hydrological Sciences.

Wallbrink, P. J., Murray, A. S., Olley, J. M., and Olive, L. J. (1998). Determining sources and transit times of suspended sediment in the Murrumbidgee River, New South Wales, Australia, using fallout 137Cs and 210Pb. Water Resources Research, 34(4), 879-887.

Wallbrink, P. J., Olley, J. M., Murray, A. S., and Olive, L. J. (1996). The contribution of subsoil to sediment yield in the Murrumbidgee River basin, New South Wales, Australia. IAHS Publications-Series of Proceedings and Reports-Intern Assoc Hydrological Sciences, 236, 347-356.

Walling, D. E. (2005). Tracing suspended sediment sources in catchments and river systems. Science of the total environment, 344(1), 159-184.

Walling, D. E., and Bradley, S. B. (1989). Rates and patterns of contemporary floodplain sedimentation: a case study of the River Culm, Devon, UK.GeoJournal, 19(1), 53-62.

Walling, D. E., and Collins, A. L. (2000). Integrated assessment of catchment sediment budgets: A technical manual. School of Geography and Archaeology, University of Exeter.

Walling, D. E., Collins, A. L., and Stroud, R. W. (2008). Tracing suspended sediment and particulate phosphorus sources in catchments. Journal of Hydrology, 350(3), 274-289.

Walling, D. E., Owens, P. N., and Leeks, G. J. (1999). Fingerprinting suspended sediment sources in the catchment of the River Ouse, Yorkshire, UK. Hydrological Processes, 13(7), 955-975.

Walling, D. E., Owens, P. N., Waterfall, B. D., Leeks, G. J., and Wass, P. D. (2000). The particle size characteristics of fluvial suspended sediment in the Humber and Tweed catchments, UK. Science of the Total Environment, 251, 205-222.

Wethered, A. S., Ralph, T. J., Smith, H. G., Fryirs, K. A., and Heijnis, H. (2015). Quantifying fluvial (dis) connectivity in an agricultural catchment using a geomorphic approach and sediment source tracing. Journal of Soils and Sediments, 15(10), 2052-2066.

Wilkinson, S. N., Hancock, G. J., Bartley, R., Hawdon, A. A., and Keen, R. J. (2013). Using sediment tracing to assess processes and spatial patterns of erosion in grazed rangelands, Burdekin River basin, Australia. Agriculture, ecosystems and environment, 180, 90-102. 